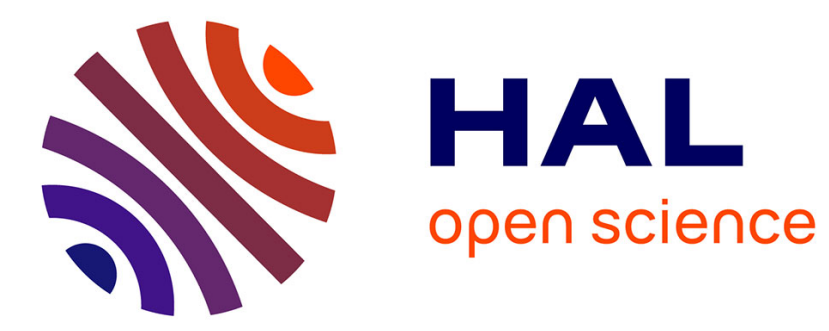

\title{
Modeling of the Coupling of Microstructure and Macrosegregation in a Direct Chill Cast Al-Cu Billet
}

Laurent Heyvaert, Marie Bedel, Miha Založnik, Hervé Combeau

\section{To cite this version:}

Laurent Heyvaert, Marie Bedel, Miha Založnik, Hervé Combeau. Modeling of the Coupling of Microstructure and Macrosegregation in a Direct Chill Cast Al-Cu Billet. Metallurgical and Materials Transactions A, 2017, 48 (10), pp.4713-4734. 10.1007/s11661-017-4238-z . hal-01709089

\section{HAL Id: hal-01709089 \\ https://hal.univ-lorraine.fr/hal-01709089}

Submitted on 14 Feb 2018

HAL is a multi-disciplinary open access archive for the deposit and dissemination of scientific research documents, whether they are published or not. The documents may come from teaching and research institutions in France or abroad, or from public or private research centers.
L'archive ouverte pluridisciplinaire HAL, est destinée au dépôt et à la diffusion de documents scientifiques de niveau recherche, publiés ou non, émanant des établissements d'enseignement et de recherche français ou étrangers, des laboratoires publics ou privés. 


\section{Modeling of the coupling of microstructure and macrosegregation in a direct chill cast $\mathrm{Al}-\mathrm{Cu}$ billet}

Laurent Heyvaert $^{1}$, Marie Bedel ${ }^{1,2}$, Miha Založnik ${ }^{1}$, Hervé Combeau ${ }^{1}$

${ }^{1}$ Institut Jean Lamour, Dept. SI2M, CNRS - Université de Lorraine, Parc de Saurupt CS 50840, F-54011 Nancy CEDEX, France

2 École Nationale Supérieure d'Arts et Métiers, Campus d'Aix-en-Provence, F-13617 Aix-en-Provence, France

* Corresponding author: miha.zaloznik@univ-lorraine.fr

\section{Abstract}

The macroscopic multiphase flow and the growth of the solidification microstructures in the mushy zone of a direct chill (DC) casting are closely coupled. These couplings are the key to the understanding of the formation of the macrosegregation and of the nonuniform microstructure of the casting. In the present paper we use a multiphase and multiscale model to provide a fully coupled picture of the links between macrosegregation and microstructure in a DC cast billet. The model describes nucleation from inoculant particles and growth of dendritic and globular equiaxed crystal grains, fully coupled with macroscopic transport phenomena: fluid flow induced by natural convection and solidification shrinkage, heat, mass, and solute mass transport, motion of free-floating equiaxed grains and of grain-refiner particles. We compare our simulations to experiments on grainrefined and non grain-refined industrial size billets from literature. We show that a transition between dendritic and globular grain morphology triggered by the grain refinement is the key to the explanation of the differences between the macrosegregation patterns in the two billets. We further show that the grain size and morphology are strongly affected by the macroscopic transport of freefloating equiaxed grains and of grain-refiner particles.

\section{Introduction}

Direct chill (DC) casting is the principal process for the production of wrought aluminum alloys. It is used to produce cylindrical billets for extrusion of profiles and rectangular ingots for rolling of plates, strip, and foil. Macrosegregation is a common defect in DC casting and can lead to nonuniform material properties. Since it is generally aggravated by higher casting speeds and casting size, it also limits productivity. Macrosegregation is characterized by variations of the chemical composition on a scale larger than the crystal grains, which cannot be mitigated during the further downstream processing. The redistribution of the chemical species (solutes) at the product scale during solidification is caused by the relative motion of liquid and solid with different compositions. It is generally admitted that the macrosegregation in DC casting is a consequence of an intricate interplay of the melt flow, induced by thermosolutal natural convection, shrinkage, and pouring, and of the transport of solute-lean free-floating grains [1-3]. Melt flow due to stress-induced deformation can also play a role [4]. The importance of the individual mechanisms varies depending on the alloy, casting shape and size and process parameters [3]. It is however difficult to construct a simplified general image of the macrosegregation formation in DC casting, because all these strongly coupled transport phenomena have a significant impact. A thorough understanding of the involved mechanisms and of their coupling is required to be able to develop robust solutions that would 
mitigate the macrosegregation [5].

The interplay of the transport mechanisms that cause macrosegregation is closely linked to the microstructure of the solidifying grains. The grain size and the grain morphology (dendritic or globular) determine the dynamics of settling of the free-floating equiaxed grains, the compactness of the packed grain layer, and its hydrodynamic permeability. They thus affect the macrosegregation induced by the settling of solute-lean grains and the expulsion of solute-rich liquid - the prime cause of the negative centerline segregation. Furthermore, the grain size and morphology influence the macrosegregation formed by permeation of solute-rich liquid through the packed layer. These effects have been clearly demonstrated in steel ingot casting, using a combination of industrial scale experiments and multiscale numerical modeling [6-10]. It was shown that globular free-floating grains lead to much more pronounced settling-induced macrosegregation than dendritic grains. Such detailed modeling has not yet been done for DC casting.

Most experimental investigations of the influence of grain motion on macrosegregation in DC casting used varying levels of grain refinement as a control process parameter, in order to stimulate or to stifle grain motion. However, the influence of grain refinement on macrosegregation is complex. On the one hand, grain refinement results into smaller grains that are more easily entrained by the liquid and have smaller slip velocities, thus reducing settling-induced macrosegregation. On the other hand it also promotes more compact globular grain morphologies that settle faster than dendritic grains of the same size and result into a larger fraction of solute-lean solid in the packed grain layer than dendritic grains. Moreover, a modification of grain size and morphology can alter the structure of the multiphase flow in the mushy zone. Due to this complexity, the experimental investigations, which were done with different alloys, casting formats and casting parameters, led to disparate conclusions and interpretations, sparking some controversy. In small 2000 series billets, no significant influence of grain refinement on macrosegregation was observed [11,12]. In large Al-Cu billets Finn et al. [13] observed that the addition of grain refiner led to a reversal from negative to positive centerline segregation, while Vreeman et al. [14] showed an increase of negative segregation. In large 5000series sheet ingots, grain refinement clearly led to more pronounced negative centerline segregation [15-19]. The grain morphology, more globular in grain-refined castings and more dendritic in non grain-refined castings, was seen as a key factor $[17,20]$. An impact of the morphology on both the motion of free-floating equiaxed grains and on the permeability of the packed grain layer was argued to be the reason for the modification of the macrosegregation patterns.

The support of modeling is vital in the investigation of the physical mechanisms that link the microstructure and the macrosegregation in a complex casting process. However, concurrent coupled simulation of microstructure and the transport phenomena in DC casting is still lacking. Past models were not able to capture these couplings because they used various simplifications. Most models were based on simple equilibrium models of phase change and lacked a description of the kinetics of the microstructure nucleation and growth [21-25]. Models that included grain growth kinetics did not account for grain motion [20] or assumed a globular grain morphology [1,26]. Detailed modeling focusing on grain structure and morphology did not consider grain motion and macrosegregation [27]. The success in predicting macrosegregation was limited to globular structures and could not correctly capture the impact of grain morphology and of grain refinement.

Recently we presented a more advanced model of DC casting, which accounts for macrosegregation with grain motion, fully coupled with nucleation on grain-refiner particles and with the kinetics of grain growth and morphology development [28,29]. This model was successful in predicting the influence of the flow on the distribution of grain size and the influence of grain refinement on the grain morphology in a large sheet ingot $[10,29]$. In the present paper we use this model to provide a 
91 fully coupled picture of the links between macrosegregation and microstructure in a DC cast billet. We use the experimental results presented by Vreeman et al. on Al-Cu billets [14] as a reference to which we compare our model predictions. The experiments were made on two grain-refined and non grain-refined billets cast under the same conditions. We perform a detailed comparison of the simulations to the experiments and a critical analysis of the coupled phenomena of macroscopic transport and microstructure formation. We show that a morphology transition triggered by the grain refinement is the key to the explanation of the differences of the macrosegregation patterns in the two billets and we show that the microstructure is strongly affected by the macroscopic transport of freefloating equiaxed grains and of grain-refiner particles.

\section{Model}

102

103

104

105

106

107

108

109

The multiscale multiphase model of solidification is based on a volume averaging method with three hydrodynamic phases [30,31], as schematically shown in Fig. 1. The model is an extension of the earlier two-phase model $[1,6,28,32]$ and uses the same constitutive relations and numerical solution methods. The two crucial extensions are the more detailed three-phase description of the grain growth kinetics and the inclusion of inertial and of solid-solid interaction forces in the momentum balance equations for the solid phase. Both extensions are detailed below and all model equations are summarized in Table 1. A detailed description of the model derivation is given in the aforementioned references.

The model couples phenomena at the microscopic and the macroscopic scales. The conservation equations for mass, solute, momentum, and the population of inoculant particles and of solid grains include terms accounting for macroscopic transport and for interphase exchange at the microscopic scale. On a microscopic scale, the dendritic grain structures are described in a volume-averaged sense by the volume fractions of the solid phase, of the grain envelopes, of the extradendritic liquid (liquid outside the envelopes), and of the interdendritic liquid (liquid inside the envelopes). The modeling of the growth kinetics of these structures is presented in Section 2.1. The motion of the liquid melt and of the solid grains at the macroscopic scale are modeled by taking into account: buoyancy-driven flow induced by liquid density variations (thermosolutal convection modeled with the Boussinesq approximation) and by the density difference between solid and liquid phases, inertial forces, drag forces at the solid-liquid interfaces, momentum transfer by collisions between grains, and fluid flow induced by solidification shrinkage. The solid grains are assumed to nucleate exclusively by athermal heterogeneous nucleation [33] on inoculant particles. A size distribution of the inoculant particles is considered and the nucleation undercooling is inversely proportional to the particle size. The distribution is discretized into classes (or bins) and population balances are used to describe the advection and the vanishing of the individual size classes [1,28]. The inoculant particles are supposed to move at the liquid velocity. This description of nucleation is suited for modeling nucleation on grain refiner particles, used in DC casting. After nucleating, the solid grains are considered as a dispersed solid phase and remain such until the local grain envelope volume fraction exceeds the packing fraction $g_{e p}$. We call this the slurry flow regime. At envelope volume fractions above packing, $g_{e}>g_{e p}$, we consider the solid phase to be stationary and the drag at the solid-liquid interfaces is described by a Darcy term modeled by the Kozeny-Carman law. We call this the porous flow regime. Note that the grain morphology plays a major role in packing. Dendritic grains, whose envelopes occupy more space than their solid skeleton, pack sooner in the solidification process (i.e. at lower solid fraction) than globular grains. 
L. Heyvaert et al., Coupling of microstructure and macrosegregation ..., Metall. Mater. Trans. A 48 (2017)
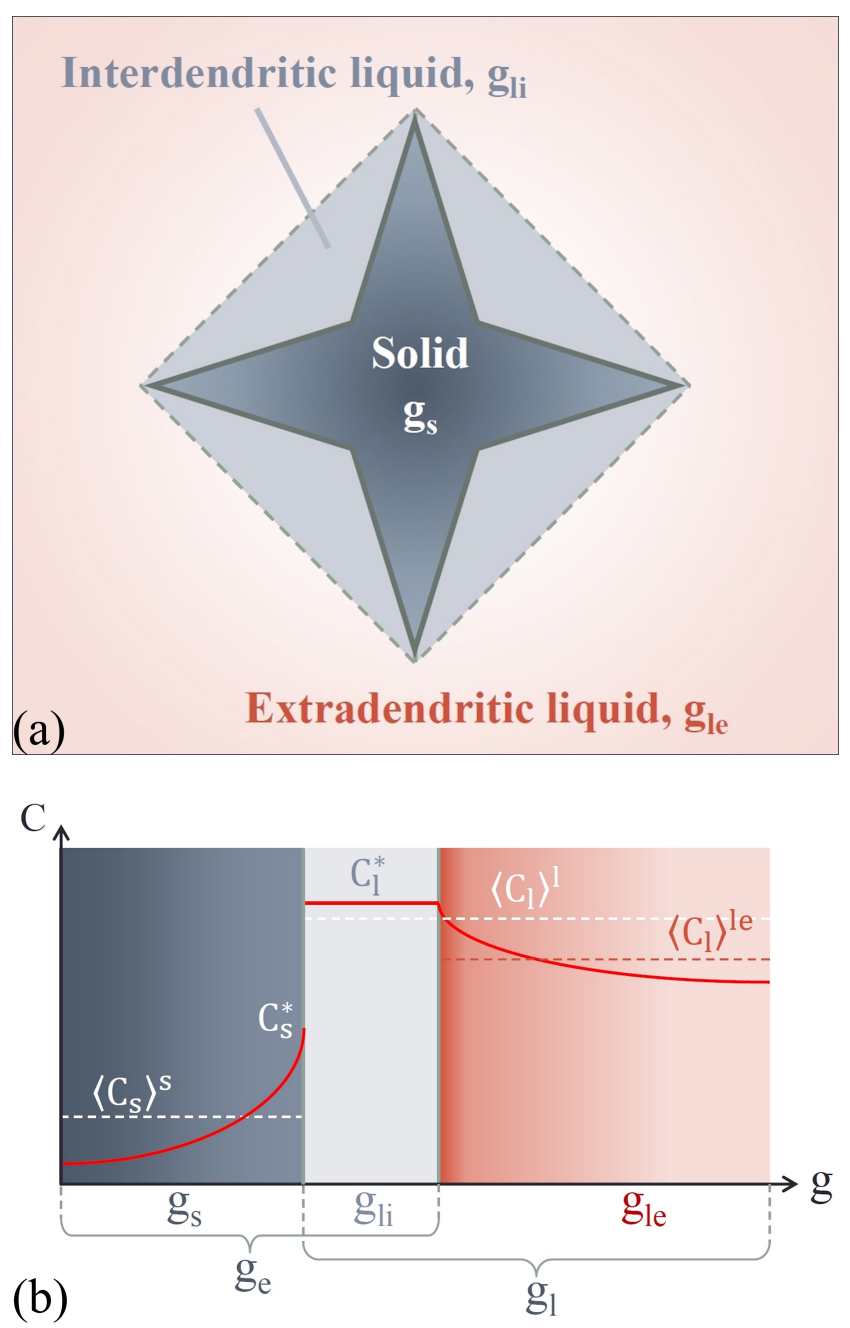

Figure 1: (a) Schematics of the hydrodynamic phases in the three-phase model. (b) Solute profiles in the three phases, solute concentrations and volume fractions. 
Table 1: Summary of the model equations

\section{Averaged conservation equations}

Total mass

conservation

$$
\frac{\partial}{\partial t}\left(g_{s} \rho_{s}+g_{l} \rho_{l}\right)+\nabla \cdot\left(g_{s} \rho_{s}\left\langle\overrightarrow{v_{s}}\right\rangle^{s}+g_{l} \rho_{l}\left\langle\overrightarrow{v_{l}}\right\rangle^{l}\right)=0
$$

Mass balance, solid phase

$$
\frac{\partial}{\partial t}\left(g_{s} \rho_{s}\right)+\nabla \cdot\left(g_{s} \rho_{s}\left\langle\overrightarrow{v_{s}}\right\rangle^{s}\right)=\Gamma_{s}+\Phi_{s}
$$

Volume balance, dendrite envelopes

$$
\frac{\partial}{\partial t}\left(g_{e}\right)+\nabla \cdot\left(g_{e}\left\langle\overrightarrow{v_{s}}\right\rangle^{s}\right)=\Gamma_{e}+\frac{\Phi_{s}}{\rho_{s}}
$$

Solute mass balance, solid phase

$$
\begin{gathered}
\frac{\partial}{\partial t}\left(g_{s} \rho_{s}\left\langle C_{s}\right\rangle^{s}\right)+\nabla \cdot\left(g_{s} \rho_{s}\left\langle C_{s}\right\rangle^{s}\left\langle\overrightarrow{v_{s}}\right\rangle^{s}\right)= \\
\Gamma_{s} C_{s}^{*}+\frac{\rho_{s} S_{v}^{s} D_{s}}{\delta_{s}}\left(C_{s}^{*}-\left\langle C_{s}\right\rangle^{s}\right)+\Phi_{s} k\left\langle C_{l}\right\rangle^{l}
\end{gathered}
$$

Solute mass balance, liquid phase

$$
\begin{gathered}
\frac{\partial}{\partial t}\left(g_{l} \rho_{l}\left\langle C_{l}\right\rangle^{l}\right)+\nabla \cdot\left(g_{l} \rho_{l}\left\langle C_{l}\right\rangle^{l}\left\langle\vec{v}_{l}\right\rangle^{l}\right)= \\
-\Gamma_{s} C_{l}^{*}+\frac{\rho_{l} S_{v}^{e} D_{l}}{\delta_{l e}} \frac{g_{l}}{g_{l e}}\left(C_{l}^{*}-\left\langle C_{l}\right\rangle^{l e}\right)-\Phi_{s} k\left\langle C_{l}\right\rangle^{l}
\end{gathered}
$$

Energy balance

$$
\begin{aligned}
& \frac{\partial}{\partial t}\left(g_{s} \rho_{s} h_{s}+g_{l} \rho_{l} h_{l}\right)+\nabla \cdot\left(g_{s} \rho_{s} h_{s}\left\langle\overrightarrow{v_{s}}\right\rangle^{s}+g_{l} \rho_{l} h_{l}\left\langle\overrightarrow{v_{l}}\right\rangle^{l}\right)=\nabla \cdot(\lambda \nabla T) \\
& h_{s}=c_{p} T h_{l}=c_{p} T+L_{f}
\end{aligned}
$$

Grain density balance

$$
\frac{\partial N_{g}}{\partial t}+\nabla \cdot\left(\left\langle\overrightarrow{v_{s}}\right\rangle^{s} N_{g}\right)=\sum_{i=1}^{m} \phi_{i}
$$

Inoculant density balance, for each

$$
\frac{\partial N_{n u c}^{i}}{\partial t}+\nabla \cdot\left(\left\langle\overrightarrow{v_{l}}\right\rangle^{l} N_{n u c}^{i}\right)=-\phi_{i}(i=1 \ldots m)
$$

class $i$

Momentum conservation, solid $\frac{\partial}{\partial t}\left(\rho_{s} g_{s}\left\langle\overrightarrow{v_{s}}\right\rangle^{s}\right)+\nabla \cdot\left(\rho_{s} g_{s}\left\langle\overrightarrow{v_{s}}\right\rangle^{s}\left\langle\overrightarrow{v_{s}}\right\rangle^{s}\right)=$ phase, only when $g_{e}<-g_{s} \nabla p+\nabla \cdot\left(\mu_{s} \nabla\left(g_{s}\left\langle\overrightarrow{v_{s}}\right\rangle^{s}\right)\right)+\rho_{s} g_{s} \vec{g}-\vec{M}_{l}^{d}+\left\langle\overrightarrow{v_{s}}\right\rangle^{s} \Gamma_{s}$ $g_{e p}$

Solid viscosity

$$
\mu_{s}=\frac{\mu_{l}}{g_{e}}\left[\left(1-\frac{g_{e}}{g_{e p}^{\max }}\right)^{-2.5 g_{e p}^{\max }}-\left(1-g_{e}\right)\right]
$$


Momentum conservation, liquid phase

Permeability

Density for the buoyancy term

$$
\begin{gathered}
\frac{\partial}{\partial t}\left(\rho_{l} g_{l}\left\langle\overrightarrow{v_{l}}\right\rangle^{l}\right)+\nabla \cdot\left(\rho_{l} g_{l}\left\langle\overrightarrow{v_{l}}\right\rangle^{l}\left\langle\overrightarrow{v_{l}}\right\rangle^{l}\right)= \\
-g_{l} \nabla p+\nabla \cdot\left(\mu_{l} \nabla\left(g_{l}\left\langle\overrightarrow{v_{l}}\right\rangle^{l}\right)\right)+\rho_{l}^{b} g_{l} \vec{g}+\vec{M}_{l}^{d}-\left\langle\overrightarrow{v_{s}}\right\rangle^{S} \Gamma_{s} \\
\text { when } g_{e}<g_{e b}: \vec{M}_{l}^{d}=\frac{3 g_{e} \rho_{l} C_{d}}{4 d_{g}}\left|\left\langle\overrightarrow{v_{l}}\right\rangle^{l}-\left\langle\overrightarrow{v_{s}}\right\rangle^{S}\right|\left(\left\langle\overrightarrow{v_{l}}\right\rangle^{l}-\left\langle\overrightarrow{v_{s}}\right\rangle^{s}\right) \\
\text { when } g_{e} \geq g_{e b}: \vec{M}_{l}^{d}=\frac{-g_{l}^{2} \mu_{l}}{K}\left(\left\langle\overrightarrow{v_{l}}\right\rangle^{l}-\left\langle\overrightarrow{v_{s}}\right\rangle^{s}\right)
\end{gathered}
$$$$
K=\frac{g_{l}^{3}}{\left(1-g_{l}\right)^{2}} \frac{l_{c}^{2}}{\pi^{2} k_{K} \tau^{2}}
$$$$
\rho_{l}^{b}=\rho_{r e f}\left[1-\beta_{T}\left(T-T_{r e f}\right)-\beta_{C}\left(\left\langle C_{l}\right\rangle^{l}-C_{r e f}\right)\right]
$$

\section{Nucleation}

Activation of inoculant class $i$
If at time $t_{0},\left(T_{m}+m\left\langle C_{l}\right\rangle^{l}\left(t_{0}\right)\right)-T\left(t_{0}\right)>\Delta T_{i}: \phi_{i}=N_{n u c}^{i} \delta\left(t-t_{0}\right)$, where $\delta$ is the Dirac delta function.

Else: $\phi_{i}=0$.

$$
\Phi_{s}=\rho_{s} V_{0} \sum_{i=1}^{m} \phi_{i}
$$

Solid mass generation due to nucleation

\section{Grain growth kinetics}

Envelope growth rate

$$
\Gamma_{e n v}=\frac{1}{\sqrt{3}} S_{v}^{e} V_{t i p}
$$

Mass balance, at the

$$
\Gamma_{s}+\Gamma_{l}=0
$$

solid-liquid interface

Solute balance, at the solid-liquid interface

$$
\begin{gathered}
\frac{\rho_{s} S_{v}^{s} D_{s}}{\delta_{s}}\left(k C_{l}^{*}-\left\langle C_{s}\right\rangle^{s}\right)+\left(\frac{1-g_{s}}{1-g_{e}}\right) \frac{\rho_{l} S_{v}^{e} D_{l}}{\delta_{e}}\left(C_{l}^{*}-\left\langle C_{l}\right\rangle^{l}\right)= \\
\Gamma_{s}(1-k) C_{l}^{*}-\rho_{l}\left(g_{e}-g_{s}\right) \frac{\partial C_{l}^{*}}{\partial t}
\end{gathered}
$$

Diffusion lengths

$$
\delta_{s}=d_{s} / 10
$$




$$
\delta_{e}=\left[\frac{2}{d_{g}}+\frac{1}{\frac{D_{l}}{V_{t i p}}\left(1-\frac{8 D_{l} / V_{t i p}}{d_{f}^{2}-d_{g}^{2}}\left[\frac{d_{g}}{2}+\frac{D_{l}}{V_{t i p}}-\left(\frac{d_{f}}{2}+\frac{D_{l}}{V_{t i p}}\right) \exp \left(-\frac{d_{f}-d_{g}}{2 D_{l} / V_{t i p}}\right)\right]\right)}\right]^{-1}:
$$

Primary arm length and equivalent

$$
R_{\text {arm }}=\left(\frac{3 g_{e}}{4 N_{g}}\right)^{1 / 3} \quad d_{g}=\left(\frac{6 g_{e}}{\pi N_{g}}\right)^{1 / 3} \quad d_{s}=\left(\frac{6 g_{s}}{\pi N_{g}}\right)^{1 / 3}
$$

diameter of envelope and of solid phase

Specific surface areas of envelope and solid

$S_{v}^{e}=4 \sqrt{3} R_{a r m}^{2} N_{v} \quad S_{v}^{s}=\frac{4}{\lambda_{2}} g_{i}^{1 / 2} g_{e}\left(1-g_{i}^{m}\right)+g_{i}^{m} S_{v}^{e}$

Thermodynamic

$$
C_{s}^{*}=k C_{l}^{*}
$$

equilibrium at the

solid-liquid interface

$T=T_{m}+m C_{l}^{*}$

\section{Paraboloidal tip model $[36,53]$}

Total tip undercooling

$$
T-T_{m}-m\left\langle C_{l}\right\rangle^{l}=\frac{L_{f}}{c_{p}} \Omega_{T}+m C_{0}\left[1-\frac{1}{1-(1-k) \Omega_{C}}\right]
$$

Dimensionless

$$
\Omega_{T}=F\left(P e_{T}, P u_{T}, \operatorname{Re}_{T}\right) \quad \Omega_{C}=F\left(P e_{C}, P u_{C}, \operatorname{Re}_{C}\right)
$$

undercooling and supersaturation of a dendrite tip with convection $[31,53]$

$$
\begin{aligned}
F(P e, P u, \mathrm{Re}) & =2 P e \int_{1}^{\infty} \exp \left[-\ln \eta+\left(1-\eta^{2}\right) P e\right. \\
& \left.-\frac{2 P u}{\mathrm{E}_{1}(\mathrm{Re})}\left[1-\eta^{2}+\ln (\eta)\left(1+\eta^{2}\right)\right]\right] d \eta
\end{aligned}
$$

$$
\begin{aligned}
& P e_{T}=\frac{R_{t i p} V_{t i p}}{2 \alpha} \quad P e_{C}=\frac{R_{t i p} V_{t i p}}{2 D_{l}} \\
& P u_{T}=\frac{R_{t i p}\left|\left\langle\vec{v}_{S}\right\rangle^{S}-\left\langle\vec{v}_{l}\right\rangle^{l}\right|}{2 \alpha} \quad P u_{C}=\frac{R_{t i p}\left|\left\langle\vec{v}_{S}\right\rangle^{S}-\left\langle\vec{v}_{l}\right\rangle^{l}\right|}{2 D_{l}} \\
& \operatorname{Re}_{T}=\frac{P e_{T}+P u_{T}}{P r} \quad \operatorname{Re}_{C}=\frac{P e_{C}+P u_{C}}{S c}
\end{aligned}
$$

Tip selection criterion

$$
R_{\text {tip }}^{2} V_{t i p}=\frac{\Gamma_{\mathrm{GT}}}{\sigma^{*}}\left[\frac{m(1-k) C_{0}}{D_{l}\left(1-(1-k) \Omega_{C}\right)}+\frac{L_{f}}{2 \alpha c_{p}}\right]^{-1}
$$


L. Heyvaert et al., Coupling of microstructure and macrosegregation ..., Metall. Mater. Trans. A 48 (2017)

\section{Hemispherical tip model [37]}

Tip velocity

$$
V_{\text {tip }}=\frac{D_{l}\left(T-T_{m}-m\left\langle C_{l}\right\rangle^{l}\right)^{2}}{\Gamma_{\mathrm{GT}}(k-1) m C_{0} \pi^{2}}
$$

137 


\subsection{Three-phase model of grain growth}

The equiaxed grains are described by their size and their morphology, which can be dendritic or globular. The description of the morphology in the volume-averaged model is based on the idea of the dendrite envelope, first proposed by Rappaz and Thévoz [34]. The solid volume fraction $g_{s}$ is distinguished from the grain envelope volume fraction, $g_{e}$, as illustrated in Fig. 1a. When a grain is dendritic, its envelope, defined in the model by a regular octahedron linking the six grain tips, is larger than the solid volume of the grain. A part of the interior of the grain envelope is therefore liquid. The model distinguishes between the liquid outside the envelopes, named extradendritic liquid, and the liquid inside the envelopes, between the dendrite arms, named interdendritic liquid. Their volume fractions are $g_{l e}$ and $g_{l i}$, respectively, and their sum is the liquid fraction $g_{l}=g_{l i}+g_{l e}$. The relationships between the different volume fractions are:

$$
g_{e}+g_{l e}=g_{s}+g_{l i}+g_{l e}=g_{s}+g_{l}=1, \quad g_{i}=\frac{g_{s}}{g_{e}}
$$

The indication of the grain morphology is given by the internal solid fraction $g_{i}$, which is the ratio between the volume of the solid and the envelope. If the internal solid fraction is close to one, the grains are considered to be globular, whereas when it is close to zero, the grains are considered to be dendritic. The final morphology of the solidified equiaxed structure is usually considered to correspond to the morphology at the instant of grain packing [31].

We assume that the interdendritic and extradendritic liquid phases move at the same flow velocity $\left\langle\overrightarrow{v_{l e}}\right\rangle^{l e}=\left\langle\overrightarrow{v_{l l}}\right\rangle^{l i}=\left\langle\overrightarrow{v_{l}}\right\rangle^{l}$ and have the same density $\rho_{l e}=\rho_{l i}=\rho_{l}$. This assumption is different from the treatment in the classical three phase model with convection [30] that accounts for the partitioning of the flow passing through and around the envelopes due to drag. Our description is not as detailed, but can be reasonable for coarse dendritic grains encountered in DC casting. It also considerably simplifies the macroscopic transport equations. The macroscopic conservation equations for the two liquid phases can be summed to a single equation for the liquid, identical to those in a two-phase model. The global liquid mass conservation equation is then:

$$
\begin{gathered}
\frac{\partial}{\partial t}\left(\rho_{l} g_{l e}+\rho_{l} g_{l i}\right)+\nabla \cdot\left(\left\langle\overrightarrow{v_{l}}\right\rangle^{l}\left(\rho_{l} g_{l e}+\rho_{l} g_{l i}\right)\right)= \\
\quad=\frac{\partial}{\partial t}\left(\rho_{l} g_{l}\right)+\nabla \cdot\left(\left\langle\overrightarrow{v_{l}}\right\rangle^{l}\left(\rho_{l} g_{l}\right)\right)=-\Gamma_{s}-\Phi_{s},
\end{gathered}
$$

where $\Gamma_{S}$ and $\Phi_{S}$ are the mass transfer rates from liquid to solid due to phase change and nucleation, respectively. Similarly, the solute conservation equation is formulated in terms of the average liquid concentration, $\left\langle C_{l}\right\rangle^{l}=\left(g_{l i}\left\langle C_{l}\right\rangle^{l i}+g_{l e}\left\langle C_{l}\right\rangle^{l e}\right) / g_{l}$.

$$
\begin{aligned}
& \frac{\partial}{\partial t}\left(g_{l e}\left\langle C_{l}\right\rangle^{l e}+g_{l i}\left\langle C_{l}\right\rangle^{l i}\right)+\nabla \cdot\left(\left\langle\overrightarrow{v_{l}}\right\rangle^{l}\left(g_{l e}\left\langle C_{l}\right\rangle^{l e}+g_{l i}\left\langle C_{l}\right\rangle^{l i}\right)\right)= \\
& =\frac{\partial}{\partial t}\left(g_{l}\left\langle C_{l}\right\rangle^{l}\right)+\nabla \cdot\left(g_{l}\left\langle\overrightarrow{v_{l}}\right\rangle^{l}\left\langle C_{l}\right\rangle^{l}\right)=\frac{1}{\rho_{l}}\left(-C_{s}^{*} \Gamma_{s}-C_{s}^{*} \Phi_{s}-j_{s}^{d}\right)
\end{aligned}
$$

where $\Gamma_{k}$ is the mass flux into phase $k$ per unit volume at the phase interface due to phase change and $\Phi_{k}$ is the phase mass transfer rate due to nucleation. Note that the total of all interfacial fluxes is zero, therefore the sum of the fluxes into both liquid phases is equal to minus the sum of the fluxes into the solid phase.

The balance equations for interphase exchange of mass and solute at the microscopic scale are modified by the three-phase description. Nevertheless, Tveito [35] derived a formulation of the equations in terms of the average liquid concentration $\left\langle C_{l}\right\rangle^{l}$ that results into equations of a form similar 
to that of the two-phase model. A detailed derivation of these equations is given in Appendix A. The interdendritic liquid is assumed to be perfectly mixed at the uniform concentration equal to the thermodynamic equilibrium concentration at the solid-liquid interface. Diffusion and convection govern the transport of solute in the extradendritic liquid around the envelope and finite diffusion is accounted for in the solid. A typical solute profile in and around a grain is illustrated in Fig. 1b.

The growth of the octahedral envelopes is driven by the growth of the primary tips corresponding to the six vertices. Analytical expressions for the dendrite tip velocity as a function of the undercooling exist for paraboloid [36] and hemispherical tip shapes [37]. A freely growing dendrite tip is accurately described by a paraboloid. However, Nielsen et al. [38] pointed out that the hemispherical tip model gave better predictions of the experimentally observed morphologies of interacting grains in inoculated Al-Cu alloys. Therefore, both tip models are considered in this study.

\subsection{Modeling of the slurry flow}

In the slurry flow regime the free-floating equiaxed grains move with a velocity that is distinct from the liquid velocity. The velocity of each phase is determined with a separate momentum balance. Because of the assumption that the interdendritic and the extradendritic liquid have the same velocity, the mass and momentum balances can be summed and written globally for the liquid. Thus, only a solid and a liquid hydrodynamic phase need to be considered. The averaged mass balance for phase $k(k=s, l)$ is given by:

$$
\frac{\partial}{\partial t}\left(g_{k} \rho_{k}\right)+\nabla \cdot\left(g_{k} \rho_{k}\left\langle\overrightarrow{v_{k}}\right\rangle^{k}\right)=\Gamma_{k}+\Phi_{k}
$$

The momentum conservation equation for phase $k$ is as follows:

$$
\begin{aligned}
\frac{\partial}{\partial t}\left(g_{k} \rho_{k}\left\langle\overrightarrow{v_{k}}\right\rangle^{k}\right) & +\nabla \cdot\left(g_{k} \rho_{k}\left\langle\overrightarrow{v_{k}}\right\rangle^{k}\left\langle\overrightarrow{v_{k}}\right\rangle^{k}\right) \\
= & -g_{k} \nabla p_{k}+\nabla \cdot\left(g_{k}\left\langle\tau_{k}\right\rangle^{k}\right)+g_{k} \rho_{k}^{b} \vec{g}+M_{k}^{d}+M_{k}^{\Gamma}+M_{k}^{\Phi}
\end{aligned}
$$

where $M_{k}^{d}$ is the interfacial drag force per unit volume, $M_{k}^{\Gamma}$ is the momentum flux across the solidliquid interface due to phase change and $M_{k}^{\Phi}$ is the momentum transfer due to nucleation of the solid from the liquid phase. The nucleation term is negligible.

The interfacial drag force is modeled as:

$$
\overrightarrow{M_{s}^{d}}=-\overrightarrow{M_{l}^{d}}=\frac{3 g_{e} \rho_{l} C_{d}}{4 d_{g}}\left|\left\langle\overrightarrow{v_{l}}\right\rangle^{l}-\left\langle\overrightarrow{v_{s}}\right\rangle^{s}\right|\left(\left\langle\overrightarrow{v_{l}}\right\rangle^{l}-\left\langle\overrightarrow{v_{s}}\right\rangle^{s}\right)
$$

where $d_{g}$ is the equivalent spherical diameter of the grain envelope, $\left\langle\overrightarrow{v_{s}}\right\rangle^{s}$ and $\left\langle\overrightarrow{v_{l}}\right\rangle^{l}$ are the intrinsic average velocities of the liquid and solid phases, and $C_{d}$ is the drag coefficient calculated as given in [6]. By using the envelope fraction, $g_{e}$, and the equivalent envelope diameter, $d_{g}$, the influence of the morphology on the drag is naturally taken into account. At a given solid fraction a dendritic grain experiences more drag than a globular grain and its slip velocity is smaller. The stress in the solid phase, generated by grain collisions, is modeled through a pseudo-fluid model [30,39]. Thus the stress term for both phases is formulated as:

$$
\nabla \cdot\left(g_{k}\left\langle\tau_{k}\right\rangle^{k}\right)=\nabla \cdot\left(\mu_{k} \nabla\left(g_{k}\left\langle\overrightarrow{v_{k}}\right\rangle^{k}\right)\right)
$$

The solid viscosity is calculated by the relation [40]: 


$$
\mu_{s}=\frac{\mu_{l}}{g_{e}}\left[\left(1-\frac{g_{e}}{g_{e p}^{\max }}\right)^{-2.5 g_{e p}^{\max }}-\left(1-g_{e}\right)\right],
$$

with the maximum envelope packing fraction, $g_{e p}^{\max }$, fixed at 0.6 , the approximate value for a close random packing of monodisperse spheres. The pressure is assumed to be identical in both phases.

The momentum conservation for the slurry zone may be rewritten for both phases as:

$$
\begin{gathered}
\frac{\partial}{\partial t}\left(\rho_{k} g_{k}\left\langle\overrightarrow{v_{k}}\right\rangle^{k}\right)+\nabla \cdot\left(\rho_{k} g_{k}\left\langle\overrightarrow{v_{k}}\right\rangle^{k}\left\langle\overrightarrow{v_{k}}\right\rangle^{k}\right)= \\
-g_{k} \nabla p+\nabla \cdot\left(\mu_{k} \nabla\left(g_{k}\left\langle\overrightarrow{v_{k}}\right\rangle^{k}\right)\right)+\rho_{k}^{b} g_{k} \vec{g}+\vec{M}_{k}^{d}+\Gamma_{k}\left\langle\overrightarrow{v_{k}}\right\rangle^{k}
\end{gathered}
$$

In the packed zone, considered as a porous medium, the solid is moving at the casting velocity and the drag force exerted on the liquid is

$$
\vec{M}_{l}^{d}=-\frac{g_{l}^{2} \mu_{l}}{K}\left(\left\langle\overrightarrow{v_{l}}\right\rangle^{l}-\left\langle\overrightarrow{v_{s}}\right\rangle^{S}\right)
$$

where $K$ is the hydrodynamic permeability of the microstructure. It is modeled by the KozenyCarman relation:

$$
K=\frac{g_{l}^{3}}{\left(1-g_{l}\right)^{2}} \frac{l_{c}^{2}}{\pi^{2} k_{K} \tau^{2}}
$$

where $l_{c}$ is the characteristic length of the porous microstructure. The Kozeny constant, $k_{K}$, and the tortuosity of the porous medium, $\tau$, depend on its morphology. Values for a packed bed of spheres were used: $k_{K}=5$ and $\tau=\pi / 6$. The resulting momentum equation for the liquid in the porous zone is:

$$
\begin{gathered}
\frac{\partial}{\partial t}\left(\rho_{l} g_{k}\left\langle\overrightarrow{v_{l}}\right\rangle^{l}\right)+\nabla \cdot\left(\rho_{l} g_{l}\left\langle\overrightarrow{v_{l}}\right\rangle^{l}\left\langle\overrightarrow{v_{l}}\right\rangle^{l}\right)= \\
-g_{l} \nabla p+\nabla \cdot\left(\mu_{l} \nabla\left(g_{l}\left\langle\overrightarrow{v_{l}}\right\rangle^{l}\right)\right)+\rho_{l}^{b} g_{l} \vec{g}-\frac{g_{l}^{2} \mu_{l}}{K}\left(\left\langle\overrightarrow{v_{l}}\right\rangle^{l}-\left\langle\overrightarrow{v_{s}}\right\rangle^{s}\right)-\Gamma_{s}\left\langle\overrightarrow{v_{s}}\right\rangle^{s}
\end{gathered}
$$

A summary of all model equations is presented in Table 1.

The conservation equations were solved with the finite volume method. An operator splitting scheme [32] was used to integrate the solute and solid mass conservation equations. The splitting scheme was extended to account for shrinkage induced flow and for transport of grain refiner particles [28]. The pressure-velocity coupling was resolved by the IPSA pressure correction algorithm for multiphase flows [41].

\section{DC casting experiments and their modeling}

\subsection{Experiments of Vreeman et al.}

Vreeman and coworkers [14] cast two binary-alloy Al-Cu billets of $450 \mathrm{~mm}$ diameter, one inoculated with Al-3Ti-1B grain refiner and one without grain refiner. The other casting parameters were identical. The casting speed was $1 \mathrm{~mm} / \mathrm{s}$ and hot-top molds were used. The macrosegregation was measured by X-ray fluorescence on 20 samples in each billet. More detailed information on the sampling was not given. The nominal composition of the two billets was slightly different with Al$6.1 \mathrm{wt} . \% \mathrm{Cu}$ for the grain refined and $\mathrm{Al}-5.9 \mathrm{wt} . \% \mathrm{Cu}$ for the non grain refined billet. In order to perform a direct comparison, the experimental data were shifted by $-0.1 /+0.1 \mathrm{wt} . \%$ to mimic an Al- 
$6 \mathrm{wt} . \% \mathrm{Cu}$ billet. The simulations were also performed with the composition Al- 6 wt.\%Cu for both billets. The main observation of the experiment was a substantially weaker centerline macrosegregation in the non grain refined billet.

For the grain refined billet two additional experimental results are available. The temperature field was characterized by inserting thermocouples into the metal during casting. The thermocouples were inserted at three radial positions and were pulled down with the billet at the casting speed. The exact three positions were at radial distances from the centerline of $r=10 \mathrm{~mm}$ ("centerline"), $r=106 \mathrm{~mm}$ ("mid-radius"), and $r=220 \mathrm{~mm}$ ("subsurface"). The uncertainty of the thermocouple measurements was $\pm 2{ }^{\circ} \mathrm{C}$. Characterizations of the size, position and shape of the mushy zone were obtained by a sudden addition of a molten Al-Si mixture. The etched macrograph of the billet section revealed the solidus front and an indication of the packing front. All experimental results are shown along with the simulations in the later sections.

Characterizations of the microstructure in terms of grain size and morphology were not reported by Vreeman et al. A grain size of $75 \mu \mathrm{m}$ used in their simulations was mentioned as representative of the typical grain size in both billets. The structure of the grain refined billet was asserted to be fully equiaxed. The structure of non grain refined billet was less clear and speculations about a possible columnar-to-equiaxed transitions were made $[14,42]$.

\subsection{Modeling of the experiments}

The billets were described by a 2D axisymmetric geometry. The computational mesh consisted of a grid of $60 \times 120$ finite-volume elements, with a finer mesh in the liquid and mushy zone and a coarser mesh in the fully solidified zone. The inlet was assumed to be across the whole cross-section and the feeding velocity was assumed to be uniform. The melt temperature at the inlet was $943.35 \mathrm{~K}$ (670.35 ${ }^{\circ} \mathrm{C}$ ), as specified in [14]. The mold height was $7 \mathrm{~cm}$. The top $1 \mathrm{~cm}$ of the mold was supposed to be thermally insulating and the heat transfer from the billet in the lower $6 \mathrm{~cm}$ of the mold was modeled by a heat transfer coefficient of $30 \mathrm{~W} / \mathrm{m}^{2} \mathrm{~K}$ and a reference coolant temperature of $291 \mathrm{~K}\left(18^{\circ} \mathrm{C}\right)$. The heat extraction by the cooling water in the direct chill zone was described by the well-established correlation of Weckman \& Niessen [43]. The thermophysical properties of the alloy are summarized in Table 2; they are the same as used in [14]. The thermal conductivity in the mushy zone is modeled as a phase-fraction weighted average of the solid and liquid conductivities. The density of the solid phase is considered as constant and a Boussinesq approximation is used for the liquid phase to account for the buoyancy forces acting on the liquid and on the free-floating grains. The variation of the densities during solidification is illustrated in Fig. 2. The densities in all mass balances are considered as constant. Thus the density difference that induces flow due to solidification shrinkage is given by a constant shrinkage coefficient of $\beta_{s l}=\left(\rho_{s}-\rho_{l, \text { ref }}\right) / \rho_{s}=0.032$. The size distribution of the grain refiner particles used in the nucleation law was taken from the measurements of Tronche for an AlTi-B grain refiner [44]. The distribution was discretized [28] into 13 classes of particles; the nucleation law is shown in Fig. 3. 
Table 2: Thermo-physical properties and model parameters

\begin{tabular}{|c|c|c|c|}
\hline Phase diagram $(\mathrm{Al}-\mathrm{Cu})$ & & Process parameters & \\
\hline Melting temperature of pure $\mathrm{Al}\left[{ }^{\circ} \mathrm{C}\right]^{1}$ & 677.8 & Casting temperature $\left[{ }^{\circ} \mathrm{C}\right]$ & 670.35 \\
\hline Nominal composition in $\mathrm{Cu}$ [wt.\%] & 6.0 & Casting speed $\left[\mathrm{m} \cdot \mathrm{s}^{-1}\right]$ & $10^{-3}$ \\
\hline Eutectic temperature $\left[{ }^{\circ} \mathrm{C}\right]$ & 547.85 & Heat transfer coefficient, insulation $\left[\mathrm{W} \cdot \mathrm{m}^{-2} \cdot \mathrm{K}^{-1}\right]$ & 0 \\
\hline Liquidus slope $\left[{ }^{\circ} \mathrm{C} \cdot \mathrm{wt} \%{ }^{-1}\right]$ & -3.425 & Heat transfer coefficient, mold $\left[\mathrm{W} \cdot \mathrm{m}^{-2} \cdot \mathrm{K}^{-1}\right]$ & 30 \\
\hline \multirow[t]{3}{*}{ Binary partition coefficient [-] } & 0.173 & Insulation height $[\mathrm{m}]$ & 0.01 \\
\hline & & Mold height $[\mathrm{m}]$ & 0.07 \\
\hline & & Reference coolant temperature $\left[{ }^{\circ} \mathrm{C}\right]$ & 18 \\
\hline Thermo-physical data & & Computational parameters & \\
\hline Specific heat $\left[\mathrm{J} \cdot \mathrm{kg}^{-1} \cdot \mathrm{K}^{-1}\right]$ & 1300 & Dimensions of the domain $(\mathrm{R} \times \mathrm{Y})[\mathrm{m} \times \mathrm{m}]$ & $0.225 \times 0.90$ \\
\hline Solid thermal conductivity $\left[\mathrm{W} \cdot \mathrm{m}^{-1} \cdot \mathrm{K}^{-1}\right]$ & 162 & Number of grid volumes, $\mathrm{R} \times \mathrm{Y}$ & $60 \times 130$ \\
\hline Liquid thermal conductivity $\left[\mathrm{W} \cdot \mathrm{m}^{-1} \cdot \mathrm{K}^{-1}\right]$ & 95 & & \\
\hline Reference density of the liquid, $\rho_{1, \text { ref }}\left[\mathrm{kg} / \mathrm{m}^{3}\right]$ & 2490 & & \\
\hline Latent heat of fusion $\left[\mathrm{J} \cdot \mathrm{kg}^{-1}\right]$ & $3.92 \cdot 10^{5}$ & & \\
\hline Thermal expansion coefficient, $\beta_{\mathrm{T}}\left[{ }^{\circ} \mathrm{C}^{-1}\right]$ & $1.17 \cdot 10^{-4}$ & & \\
\hline Solutal expansion coefficient, $\beta_{\mathrm{C}}\left[\mathrm{wt} \%^{-1}\right]$ & $-7.3 \cdot 10^{-3}$ & & \\
\hline Reference temperature for density $\left[{ }^{\circ} \mathrm{C}\right]$ & 660 & & \\
\hline Reference $\mathrm{Cu}$ concentration for density & 6.0 & & \\
\hline Dynamic viscosity $[\mathrm{Pa} \cdot \mathrm{s}]$ & $1.28 \cdot 10^{-3}$ & & \\
\hline Diffusion coefficient, liquid $\left[\mathrm{m}^{2} \cdot \mathrm{s}^{-1}\right]$ & $5.66 \cdot 10^{-9}$ & & \\
\hline Diffusion coefficient, solid $\left[\mathrm{m}^{2} \cdot \mathrm{s}^{-1}\right]$ & $5.6 \cdot 10^{-13}$ & & \\
\hline Shrinkage coefficient $\beta_{\mathrm{sl}}=\left(\rho_{\mathrm{s}}-\rho_{\mathrm{l}, \mathrm{ref}}\right) / \rho_{\mathrm{s}}$ & 0.0319 & & \\
\hline Characteristic length for permeability $[\mathrm{m}]$ & $7.5 \cdot 10^{-5}$ & & \\
\hline Gibbs-Thompson coefficient $[\mathrm{K} \cdot \mathrm{m}]$ & $1.9 \cdot 10^{-7}$ & & \\
\hline
\end{tabular}

${ }^{1}$ Etrapolation of the linearized liquidus line to zero concentration. 


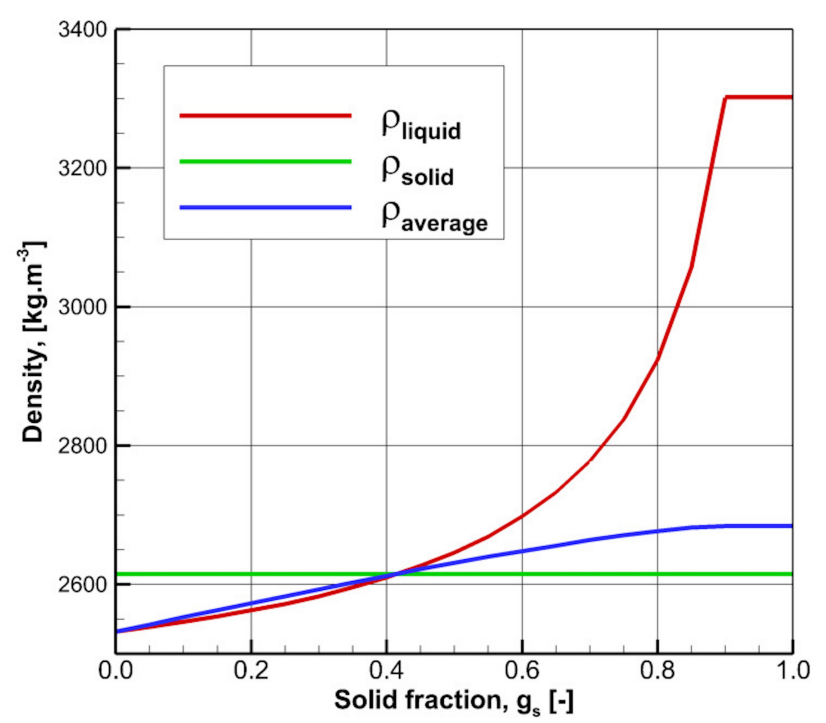

Figure 2: Evolution of solid, liquid and average densities of the alloy Al-6wt.\%Cu with solid fraction. The solidification path is assumed to follow the Scheil-Gulliver model. The variation of the solid density due to the appearance of a secondary phase upon the eutectic reaction is not accounted for.

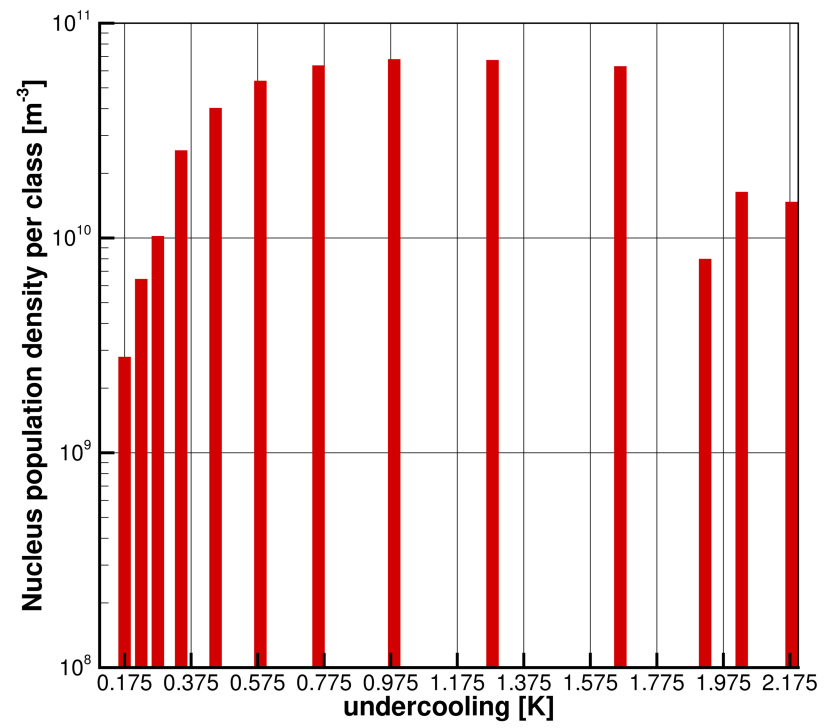

Figure 3: Discretization of the distribution of nucleation sites with respect to the critical undercooling. The distribution corresponds to the size distribution of TiB 2 particles for $1 \mathrm{~kg} / \mathrm{t}$ of Al5 Ti-1B grain refiner [28,44].

Some of our results are compared to the simulations of Vreeman et al. [14], who used a much simpler model, especially with respect to the microstructures. Their model considered the equiaxed grains to be globular and of a constant size (fixed to $75 \mu \mathrm{m}$ ), and described the solidification by the lever rule. Notably, with these assumptions the local grain population density must be adjusted in order to correspond to the solid fraction obtained from the lever rule and to the fixed grain size. Vreeman et al. used a similar criterion as the present work for packing of the grains. The grains were considered to be packed above a solid fraction of 0.3 . 


\subsection{General features of the heat transfer, flow and microstructure}

Model predictions of heat transfer, fluid flow, motion of the equiaxed grains and of solidification in the sump of the billet are illustrated in Fig. 4. These are results from a simulation that used a packing fraction of $g_{\mathrm{ep}}=0.20$ and the paraboloidal dendrite tip model.

The temperature and the solid fraction fields are shown in Figs. $4 \mathrm{a}$ and $4 \mathrm{~b}$, respectively. The radial heat extraction from the metal that is fed into the mold from the top in liquid state creates the characteristic roughly triangular shape of the solidification zone (sump). The heat extraction in the mold is relatively weak due to the particular mold design. The ingot is cooled mainly by the direct contact with the water, which starts abruptly below the mold. This explains why the sump in the rapidly cooled zone at the billet surface bends perpendicularly to the surface. In the billet center the temperature gradients are much smaller and a large part of the mushy zone in the core has a virtually homogeneous temperature that is only slightly below the liquidus temperature of $930.25 \mathrm{~K}(657.25$ $\left.{ }^{\circ} \mathrm{C}\right)$.

The flow is driven by thermosolutal natural convection and by the settling equiaxed grains. Due to the density relations in the Al-6 wt $\% \mathrm{Cu}$ alloy (Fig. 2), all three driving forces cooperate. The colder, solute enriched liquid with a higher fraction of solid grains, forming at the slopes of the sump, has a higher density than the hotter solute-lean liquid in the core. The buoyancy forces induce a descending current along the mushy zone and develop a flow in a roughly triangular pattern with one major loop, as shown in Fig. 4c. More precisely, below the inlet the flow deflects horizontally towards the mold and then rushes downwards parallel to the packing front towards the billet center. The flow velocity is the largest in the current descending along the packing front, due to the conjunction of strong buoyancy forces and drag forces induced by grain sedimentation (as the solid phase is heavier than the surrounding liquid). Then the flow slowly turns upward in the central part of the billet and runs again towards the mold.

The field of the intrinsic velocity of the solid phase is shown in Fig. 4d. The solid forms as equiaxed grains nucleating on grain refiner particles. The nucleation zone is located adjacent to the inclined part of the packing front $[1,45]$, which is a result of a particular competition between nucleation, growth and grain transport [28]. The solid grains are heavier than the liquid and tend to sediment until packing. The packing is modeled by a limit envelope volume fraction. In the example shown in Fig. 4 the packing fraction is $g_{e p}=0.2$ and the packing front is shown by a black line. The grains descend towards the billet center in a current parallel to the packing front. Some of them pack on the way, most of them accumulate in a wide packing layer at the bottom of the sump in the billet center, and some grains are dragged upwards by the liquid flow. This migration of the solid phase from the periphery to the billet core results in a global advection of latent heat in the inverse direction. This cools down the core and also produces the isothermal zone in the core of the sump. 


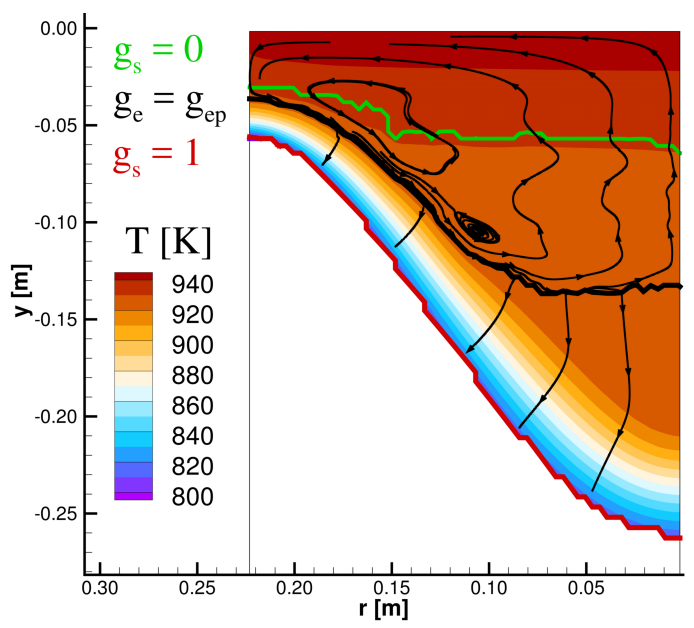

(a) Temperature

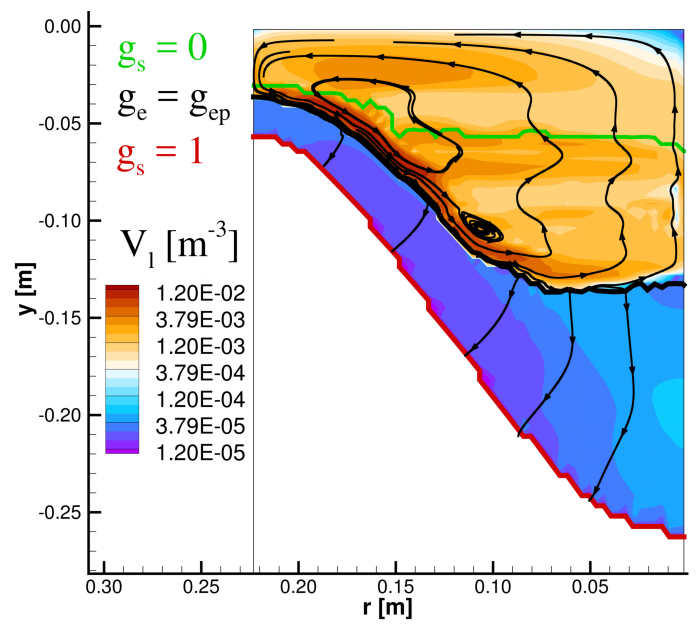

(c) Liquid velocity

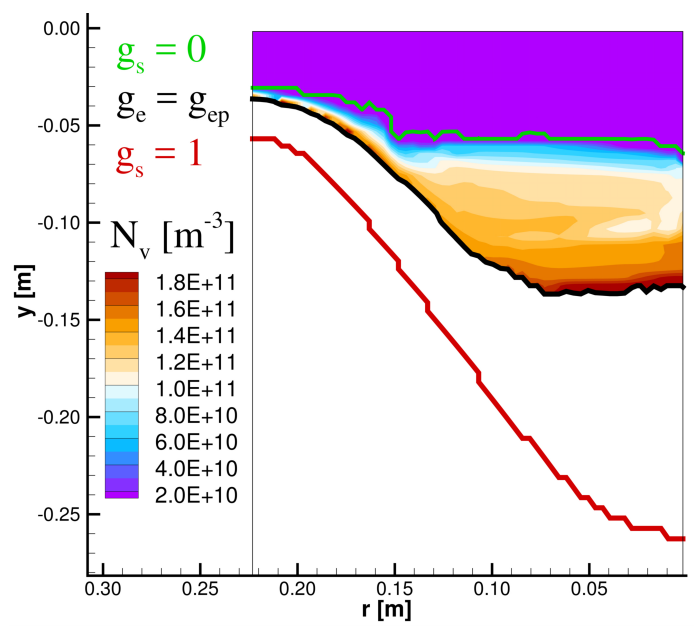

(e) Grain density

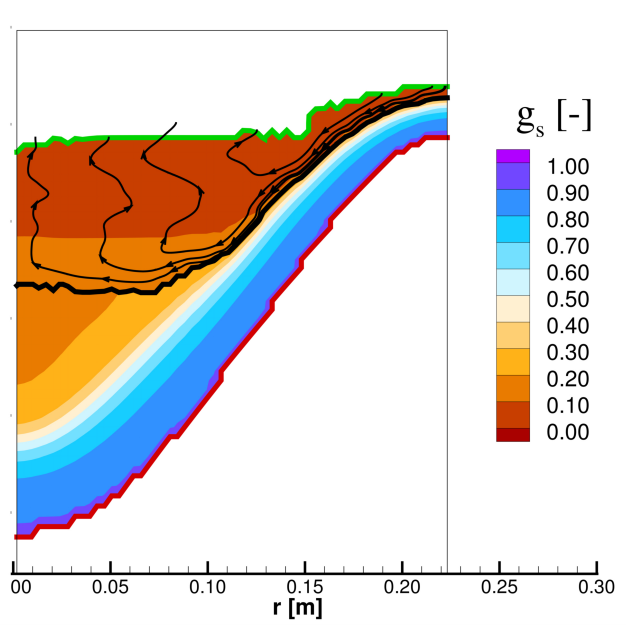

(b) Solid fraction

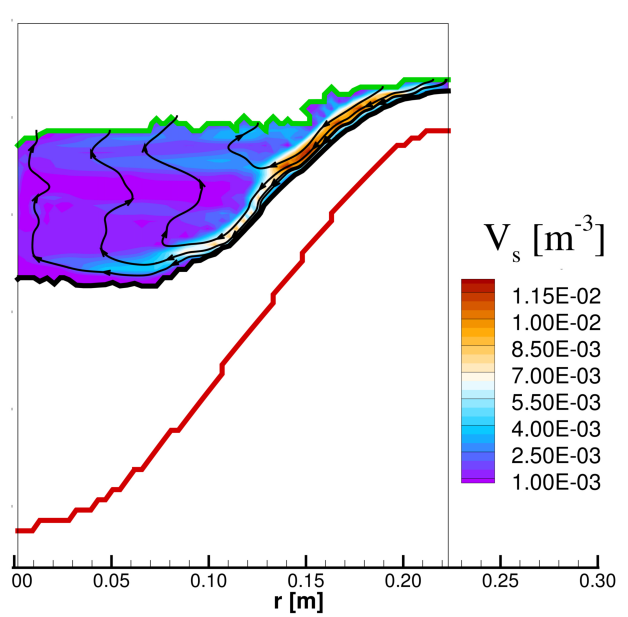

(d) Solid velocity

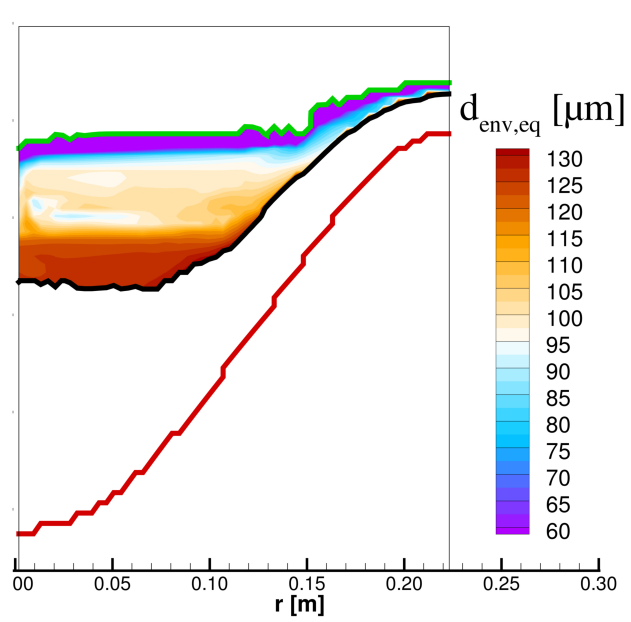

(f) Equivalent grain diameter

Figure 4: Heat transfer and flow in the billet: (a) temperature field, (b) solid fraction, (c) norm of the liquid velocity and liquid velocity streamlines, (d) norm of the solid velocity and solid velocity streamlines. The grain refined billet simulated with a packing fraction of $g_{e p}=0.2$ and the paraboloidal dendrite tip model is shown. The relative velocities are shown in the reference frame of the moving product. 
Although no information on the grain size was reported in the experiments, it is interesting to have a look to the at the distributions of grain density and grain size in the slurry zone, shown in Fig. 4e and $4 \mathrm{f}$, respectively. The variations of both grain size and grain density are mainly in the vertical direction and the highest values of both are located at the packing front. With the exception of a thin layer close to the liquid region, in most of the slurry region the grain density varies from around $8 \cdot 10^{10}$ to $1.8 \cdot 10^{11}$ grains $/ \mathrm{m}^{3}$ and the grain size from around 75 to $130 \mu \mathrm{m}$. These variations are not exceedingly large and they can justify the assumption of a fixed grain size that was adopted by Vreeman et al. [14]. The main limitation of such an assumption is that the grain size becomes an input parameter of the model that is difficult to determine a priori.

The microstructure was simulated with two different dendrite tip models, leading to significantly different predictions of the equiaxed grain morphology. Fig. 5 shows the internal solid fraction in the mushy zone obtained with both tip models. A packing fraction of $g_{e p}=0.2$ was used in both simulations. The paraboloidal tip model predicts fully globular grains in the whole mushy zone, i.e. a globular morphology from nucleation until packing of the grains. Contrarily, the hemispherical model predicts a rapid dendritization of the grains and an evolution of the morphology. Nevertheless, the grains remain dendritic until they pack. The internal solid fraction at the packing front, $g_{s i}$, is characteristic of the final grain morphology. The predicted morphology profiles across the billet radius in terms of this criterion are shown in Fig. 6. The profiles are given for simulations with both dendrite tip models and using envelope packing fractions between 0.10 and 0.55 . The plot clearly shows that the parabolic tip model predicts globular grains and the hemispheric tip model predicts dendritic grains. The latter model also indicates somewhat less pronounced dendritic morphologies in the core than at the billet surface as an overall trend. The predictions depend weakly on the packing fraction, with the tendency of giving somewhat more globularized morphologies at higher packing fractions, especially in the region that coincides with the most inclined part of the packing front. The tendency of the hemispherical tip model to predict a more pronounced dendritization of the grains than the parabolic tip model has already been reported in literature [38].
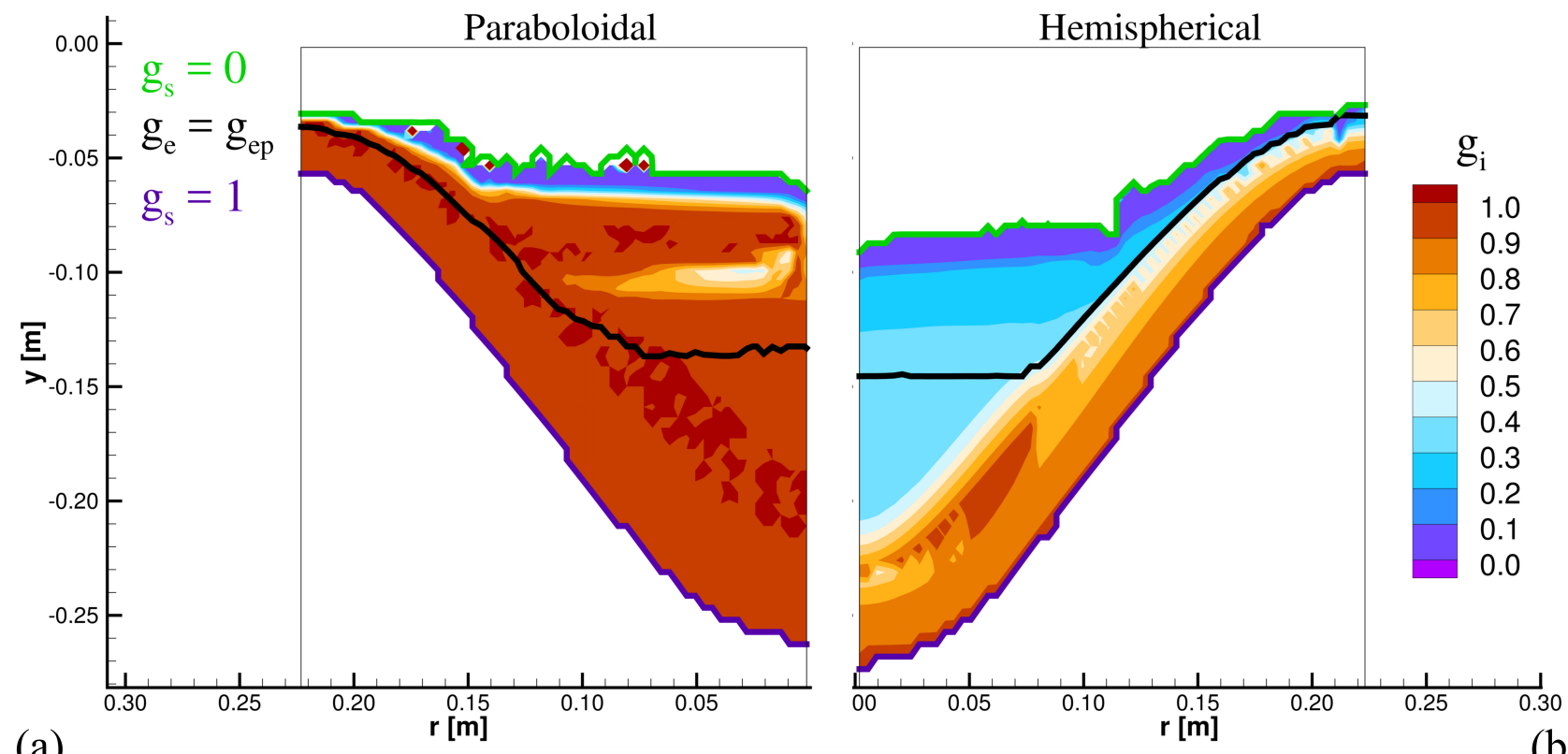

(a)

Figure 5: Internal solid fraction in the mushy zone obtained with both dendrite tip models: (a) paraboloidal, (b) hemispherical tip model. The packing fraction is $g_{e p}=0.20$ in both cases. 


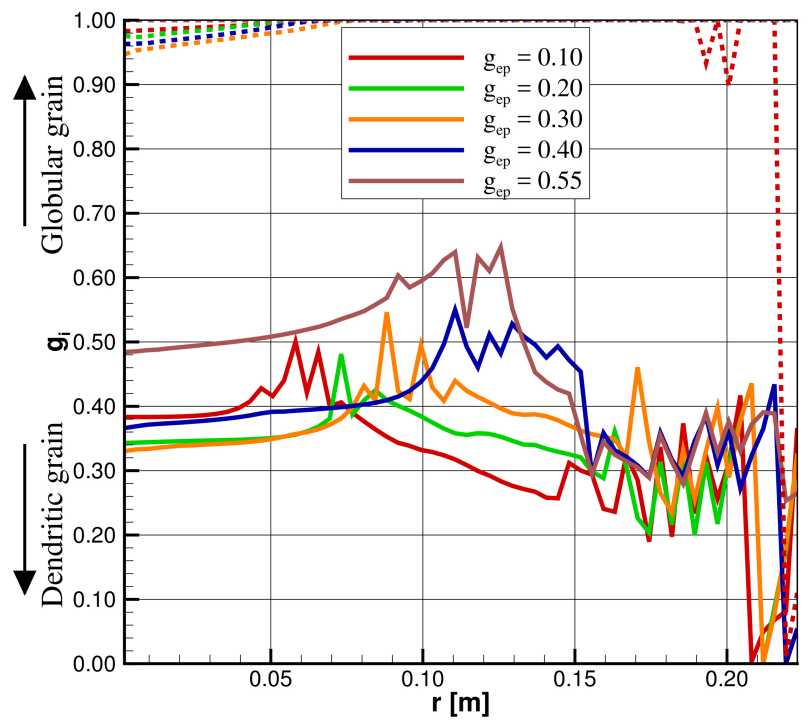

Figure 6: Internal solid fraction at packing $\left(g_{\mathrm{ip}}\right)$ for both tip growth models, hemispherical (solid lines) and paraboloidal (dashed lines).

\subsection{Comparison to measurements of temperature and sump shape}

Before analyzing the model predictions in more detail, it is essential to validate the computed thermal field by comparison to the experiment. The comparison between the experimental temperature profiles and the present model (using $g_{\text {ep }}=0.20$ and the paraboloidal tip model), as well as with the model of Vreeman et al. [14] is shown in Fig. 7a. The agreement between the experimental and the predicted profiles is very good in the center of the ingot and at mid-radius. The subsurface profile is also accurately predicted and globally fits better to the experiment than the simulations of Vreeman et al., despite the use of the generic Weckman-Niessen thermal boundary condition instead of a fit to the measurements. The centerline temperature profiles indicate that the mushy zone is nearly isothermal up to a depth of $20 \mathrm{~cm}$ in the ingot center. This isothermal zone reaches down to more than half way through the packed mushy layer (and does not end at the packing front as suggested in [14]).

The effect of the packing fraction on the thermal field is illustrated in Figs. $7 \mathrm{~b}$ and $7 \mathrm{c}$, for the paraboloidal and the hemispherical tip model, respectively. An increase of the packing fraction leads to a significant temperature decrease in the core of the billet, especially at the centerline. When the grains pack at a higher volume fraction, a larger proportion of the solid in the mushy zone is mobile. Because of this, the latent heat transport from the billet core to the periphery, induced by grain motion in the opposite direction, is intensified. This effect is more significant when the grains are globular (Fig. 7b) than when they are dendritic (Fig. 7c). The reason is that the solid fraction of globular grains at packing is higher than that of dendritic grains by a factor of $g_{\mathrm{ip}}{ }^{-1}$ and therefore a larger amount of solid is set in motion. 


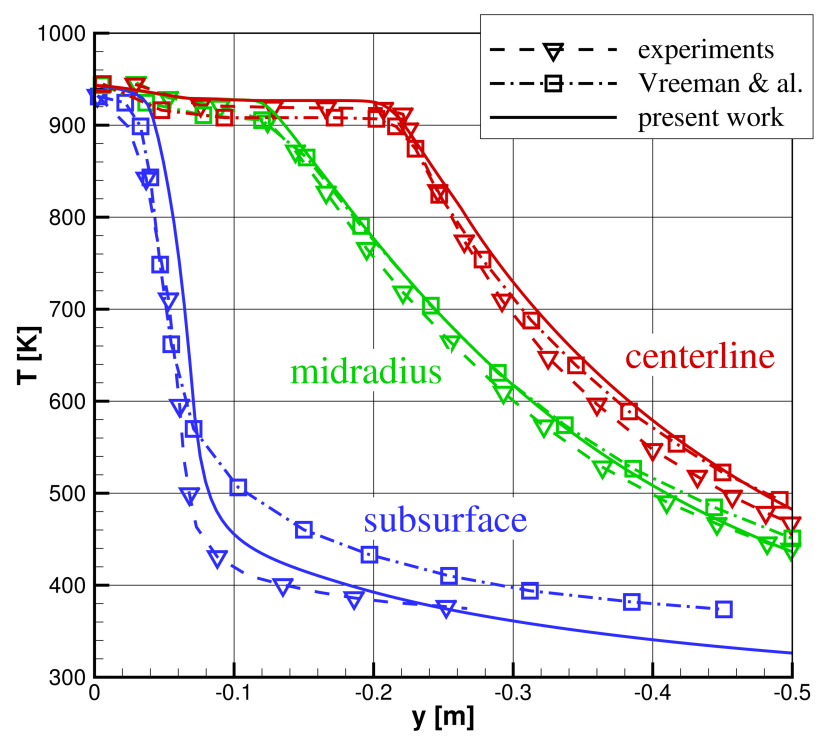

(a) Experiments and simulation

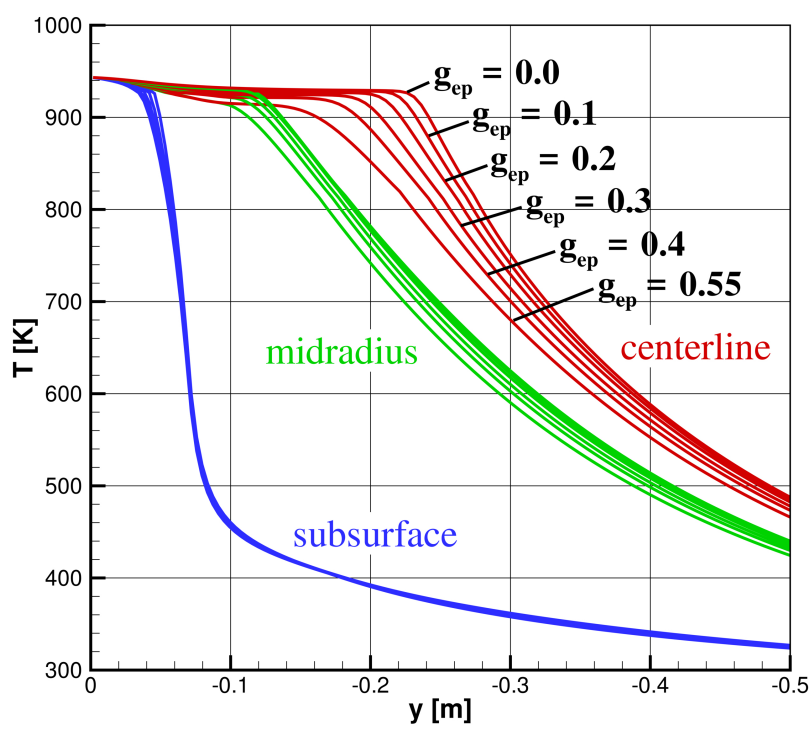

(b) Globular grains

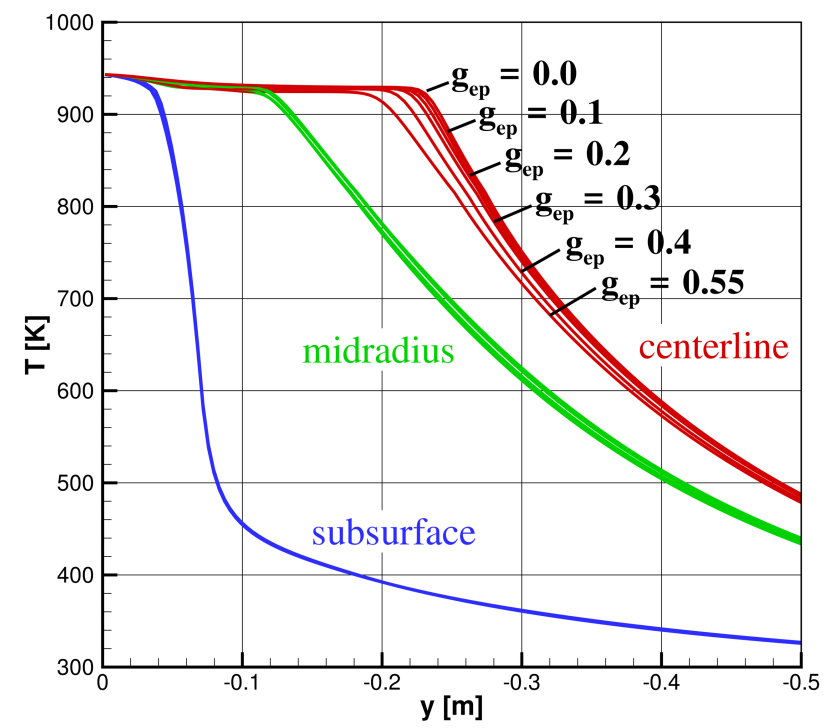

(c) Dendritic grains

Figure 7: Comparison of measured and computed vertical temperature profiles at three different radius in the ingot (subsurface in blue, midradius in green and centerline in red). (a) Comparison of simulations with globular grains (paraboloidal tip model) and $g_{e p}=0.2$ with experiments and with simulations of Vreeman et al. [14]. (b) Influence of the packing fraction on the temperature profiles for globular grain morphology (paraboloidal tip model). (c) Influence of the packing fraction on the temperature profiles for dendritic grain morphology (hemispherical tip model).

A comparison between experimental and simulated sump geometries is shown in Fig. 8. In Fig. 8a the sump shape measured in the refined billet is superimposed on the packing and the solidus fronts predicted by the model (using $g_{\mathrm{ep}}=0.20$ and the paraboloidal tip model, which gave the most accurate results). The data are shifted such that the solidus fronts are aligned at the centerline. We can see that the fit is very good, both in terms of shape and overall dimensions. In order to determine the best value of $g_{\text {ep }}$ by comparison with the experiment, two characteristic dimensions of the sump were 
extracted from the experimental measurements. The distance between the packing front and the solidus at mid-radius, $L 1$, represents the thickness of the mushy zone $\left(L 1_{\exp }=5 \mathrm{~cm}\right)$ and $L 2$ is the height of the solidus front $\left(L 2_{\text {exp }}=19.3 \mathrm{~cm}\right)$. In Fig. $8 \mathrm{~b}$ these two dimensions from a series of simulations using both dendrite tip models and a wide range of packing fractions are compared to the experimental values. We can see that the solidus height $L 2$ is well estimated overall. It decreases with increasing packing fraction. This is a consequence of an enhancement of the cooling of the core through latent heat transport by grain motion, as explained earlier. This effect is more pronounced with globular grains, as predicted by the paraboloidal tip model, than with dendritic grains, predicted by the hemispherical tip model. The prediction of the mushy zone thickness $L 1$ is much more sensitive to the packing fraction. The simulations give rather accurate results for small packing fractions $\left(g_{\text {ep }}\right.$ $=0.10-0.20)$. For larger packing fractions, the mushy zone thickness at mid-radius is clearly overestimated. The main reason is that at higher packing fractions (between 0.30 and 0.40 ) the plateau of the packing front in the core widens and its characteristic inflection shifts further outwards (compare Figs. 9a,c to Figs. 9b,d). This clearly indicates that the extent of the packed layer is overestimated when using high packing fractions. The predicted increase of the packing layer with an increasing packing fraction is stronger with the hemispherical tip model.
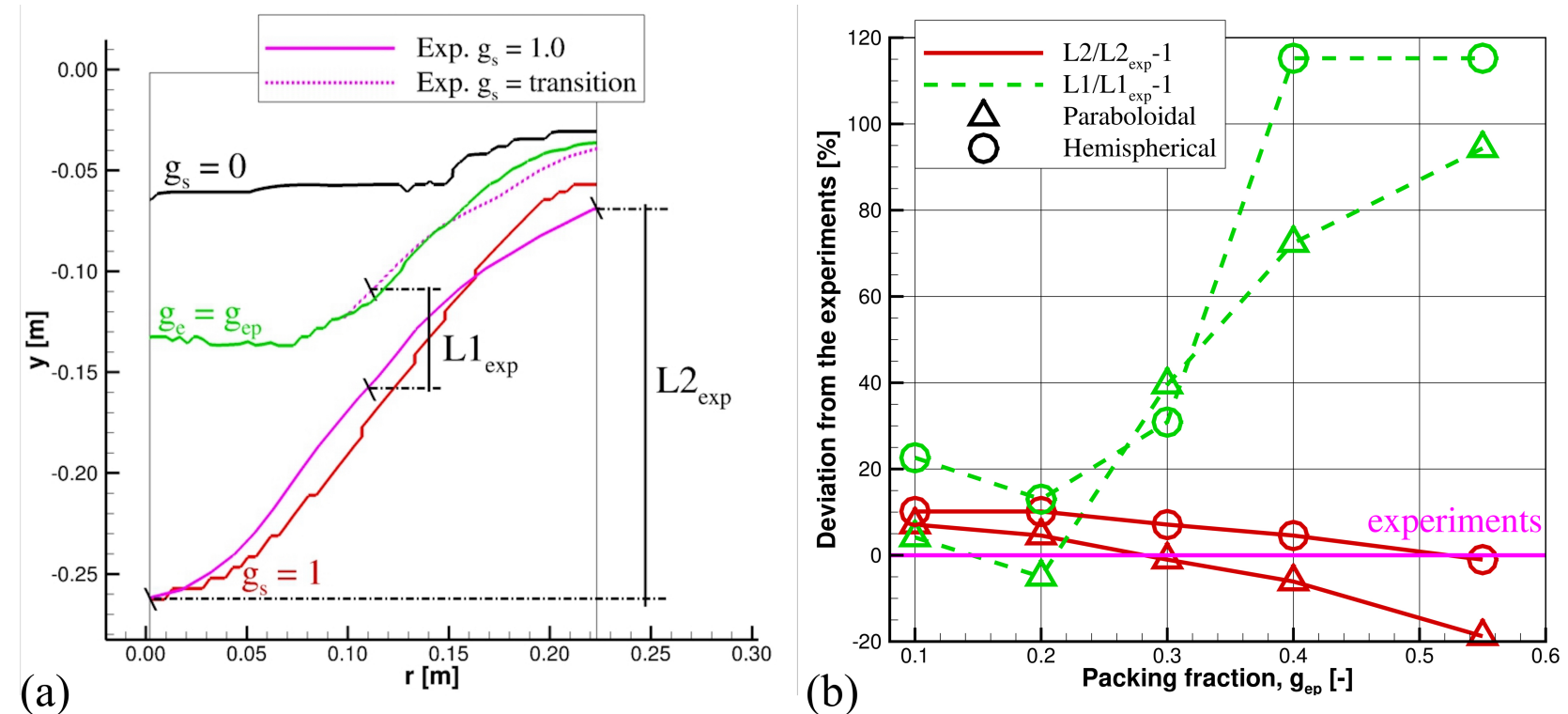

(b)

Figure 8: (a) Comparison of the sump shape obtained with the paraboloidal tip growth model and $g_{e p}=0.20$ to the experimental sump shape. (b) Relative error of the lengths L1 and L2 as a function of the packing fraction, shown for simulations with both tip models.

The validation of the model by comparison to measurements of temperature in the billet and to experimentally determined sump shape and dimensions has shown that the model predictions significantly depend on the packing fraction of the free-floating equiaxed grains. The packing fraction determines the intensity of the heat transfer due to the transport of latent heat by grain motion in the sump. This effect is particularly strong when the grains are globular due to a larger proportion of moving solid in the mushy zone. Dendritic grains have a considerably weaker influence on the heat transfer and less impact on the thermal state in the casting. 


\subsection{Macrosegregation in the grain refined billet}

The formation of the macrosegregation in the billet is shown in Fig. 9 for four cases: with globular and dendritic grain morphologies (stemming from the use of the two dendrite tip models), each for two packing fractions $\left(g_{\text {ep }}=0.20\right.$ and $\left.g_{\text {ep }}=0.40\right)$. The field of the average "mixture" composition, $C_{m}=\left(1-g_{s}\right)\left\langle C_{l}\right\rangle^{l}+g_{s}\left\langle C_{s}\right\rangle^{s}$, and the streamlines of the liquid velocity are represented. We can distinguish four zones of segregation formation [1]:

- the slurry zone, where a natural convection flow caused by thermosolutal buoyancy and grain motion determines the solute transport;

- the packing front, where the sudden accumulation of solute-lean grains causes a negative jump of the solute concentration;

- the moderately permeable packed layer (moderate $g_{1}$ ), where the natural convection and the shrinkage-induced flow determine the path of the liquid and the segregation caused thereby;

- the weakly permeable packed layer (low $g_{1}$ ), where shrinkage flow dominates the solute transport, leading to a negative segregation tendency in the center and positive at the periphery.

The coupling and superposition of these phenomena, the roots of the final macrosegregation pattern, were investigated in detail in $[1,45]$. It was shown that the grain transport is not only directly responsible for the creation of the negative centerline segregation, but also changes the sump shape and thus modifies the trajectory of the natural convection and shrinkage flow and the segregation they cause. The cooperation of the listed transport phenomena results in a radial segregation profile, shown in Fig. 10. The predicted profile is qualitatively similar to the experiment and both show a strong negative segregation in the billet center, a weak positive segregation from the mid-radius outwards, and a neutral or slightly negative segregation at the surface of the billet.

Figs. 9 and 10 show that the shape and the intensity of the predicted macrosegregation are strongly dependent on the morphology of the equiaxed grains. To a smaller, but significant degree the predicted segregation also depends on the packing fraction used in the computations. The segregation in case of globular morphologies is systematically stronger than in case of dendritic morphologies. The most striking difference is a stronger negative centerline segregation. The negative centerline segregation is favored by settling of solute-lean grains and expulsion of solute-rich liquid. If the grains have a globular morphology, they settle into a compact packing, carrying a large volume of solutelean solid phase. In comparison, ramified dendritic grains create a loose packing, with a smaller volume of solid phase replacing the solute-rich liquid. Supposing that the globular and dendritic grains pack at the same envelope fraction, the ratio of solid fraction in the packed layer between dendritic and globular grains is of the order of $g_{\text {ip }}$, the internal solid fraction of the dendritic grains at the packing front. The difference in the segregation induced by settling can be seen in the average concentration of the sedimentation layer at the packing front, which is much lower in the case of globular grains (Fig. 9a) than in the case of dendritic grains (Fig. 9b). 

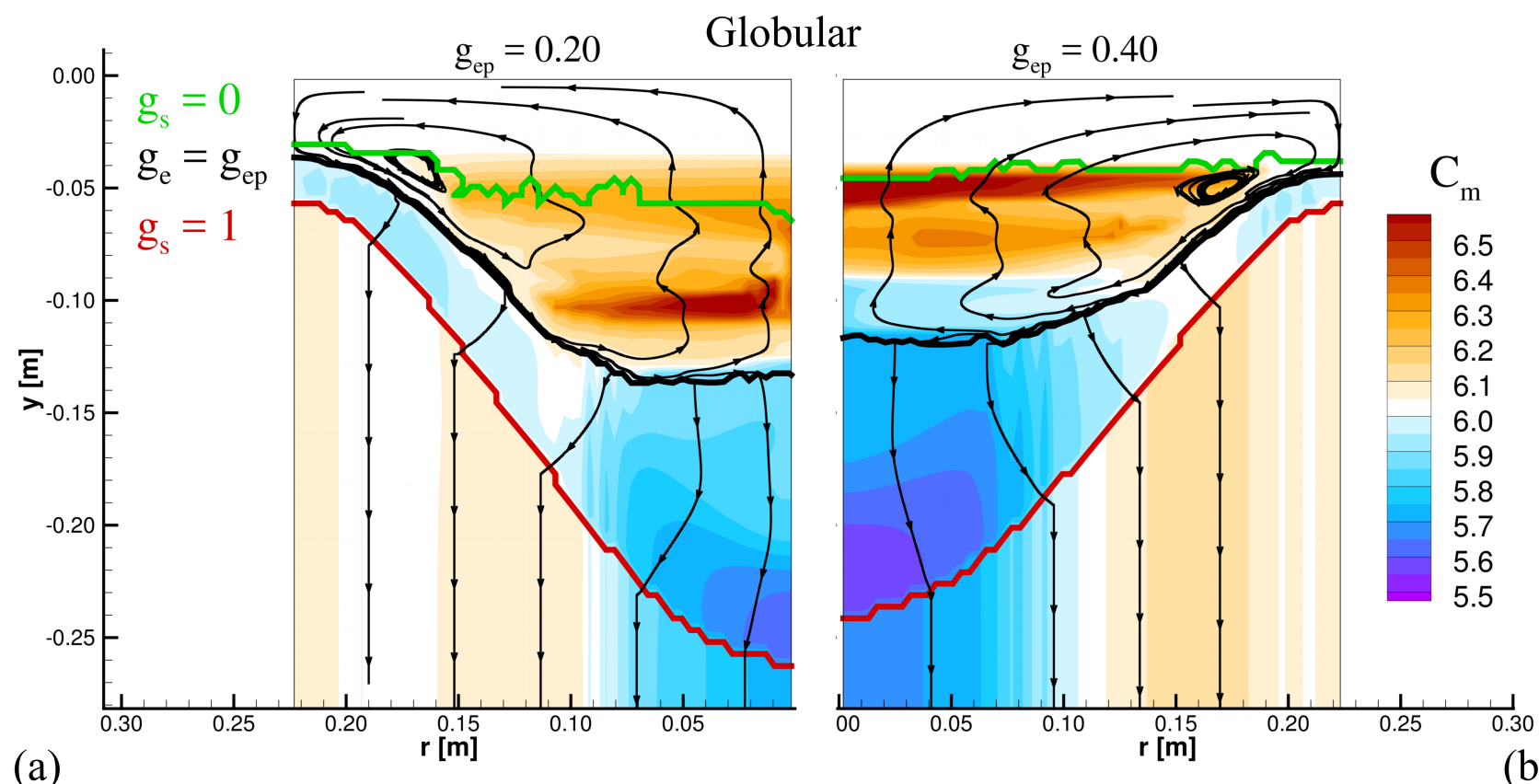

(a)
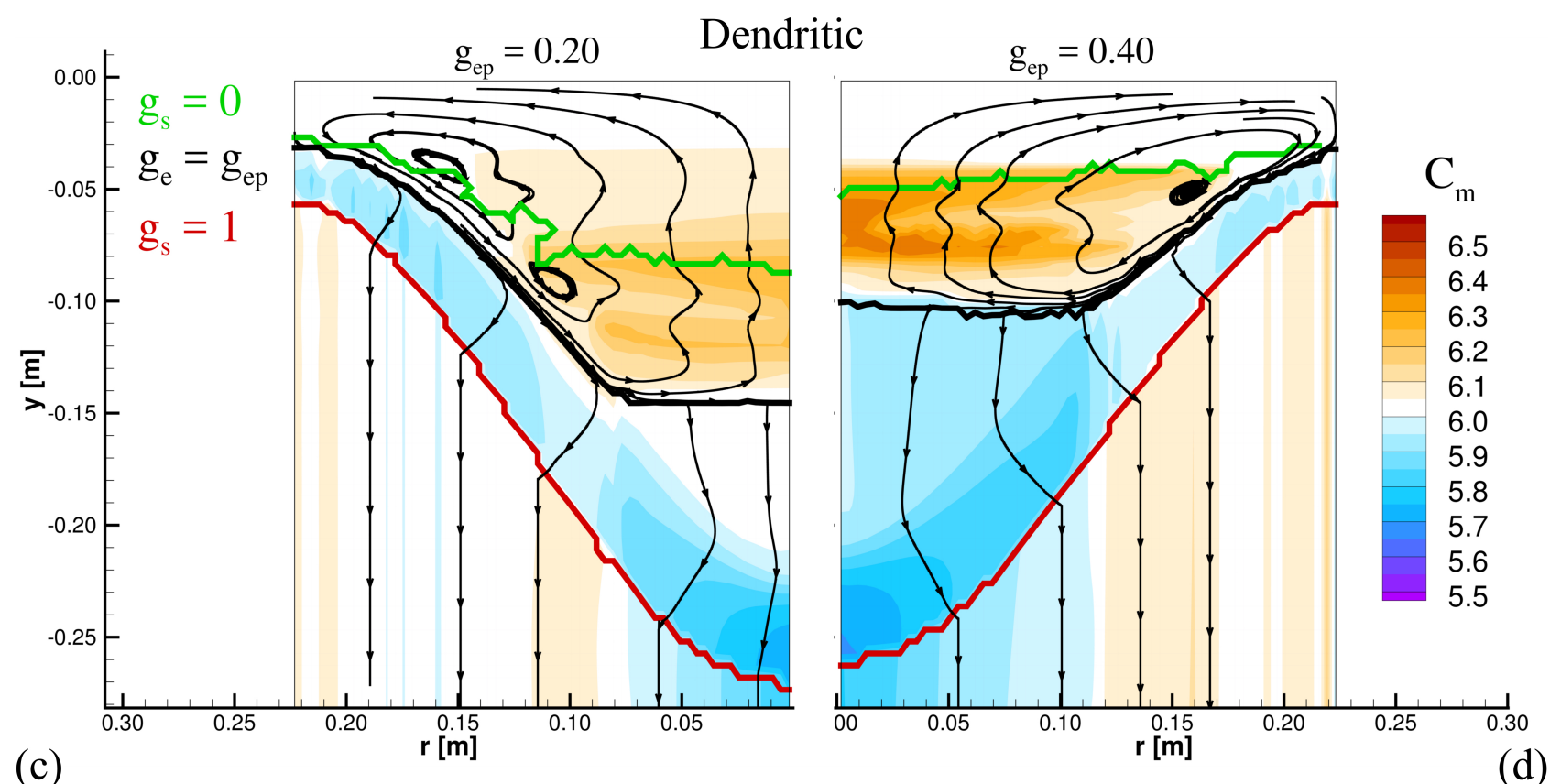

Figure 9: Macrosegregation of copper in the billet. Fields of average concentration for packing fractions of $g_{e p}=0.20$ and $g_{e p}=0.40$ and both dendrite tip models: (a) paraboloidal tip model and (b) hemispherical tip model. 


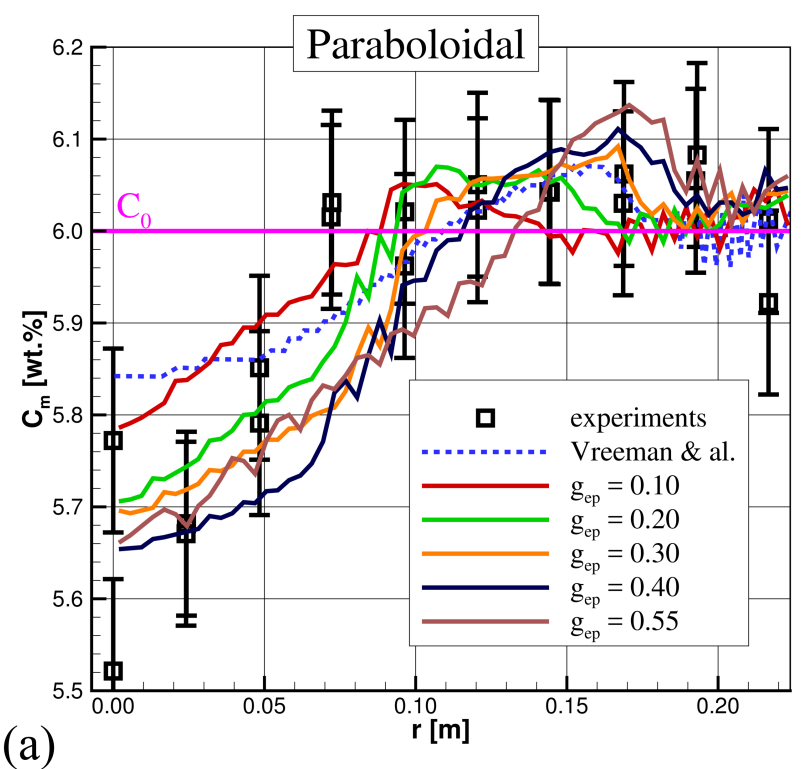

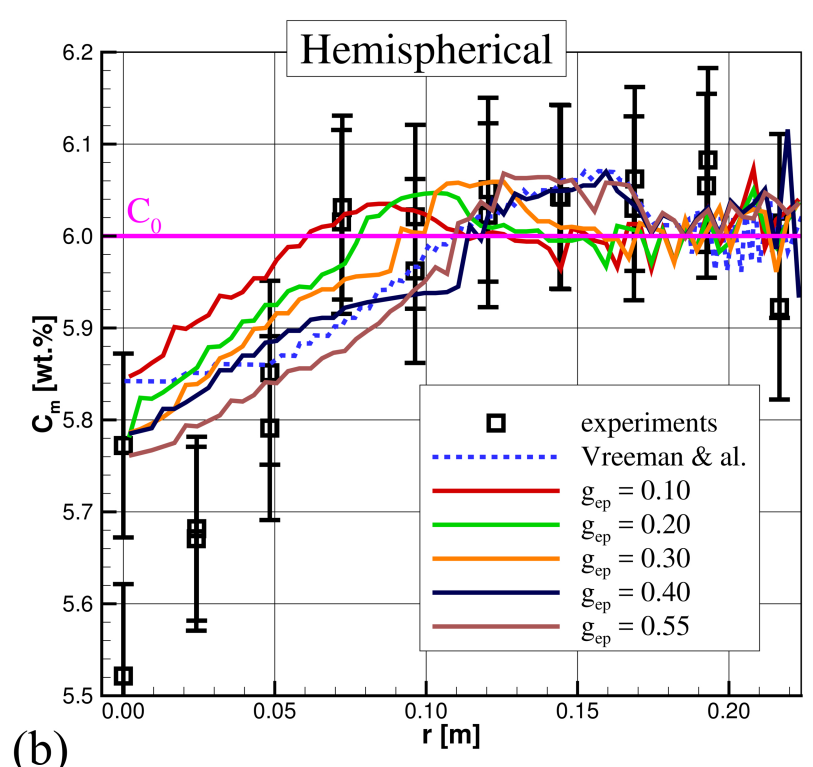

(b)

Figure 10: Macrosegregation in the grain-refined billet. Profiles of copper concentration from simulations with different packing fractions and both dendrite tip models: (a) paraboloidal tip model and (b) hemispherical tip model. The model predictions are compared to the experimental profiles and to simulations of Vreeman et al. [14].
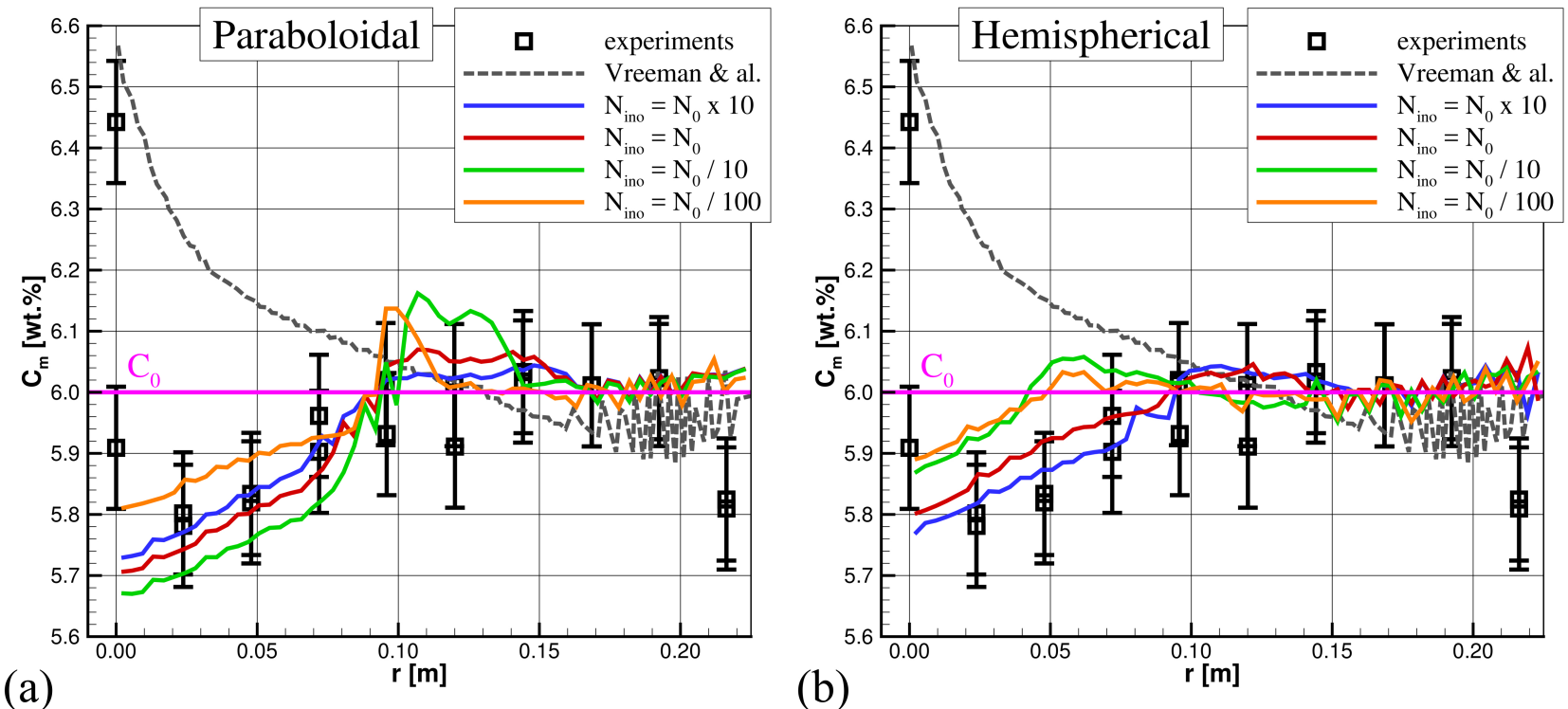

Figure 11: Macrosegregation in the non grain-refined billet. Profiles of copper concentration from simulations with different nucleation densities and both dendrite tip models: (a) paraboloidal tip model and (b) hemispherical tip model. The model predictions are compared to the experimental profiles and to simulations of Vreeman et al. [14]. $N_{0}$ refers to the distribution of nucleation sites shown in Fig. 3. 
A further effect of the morphology is the drastic influence on the hydrodynamic permeability of the packed layer. The more closely packed globular grains result in a packed layer with a solid fraction of $g_{\text {ep. }}$ This packed zone is less permeable than the loosely packed layer of dendritic grains, whose solid fraction is lower, i.e., $g_{\text {ip }} g_{\text {ep }}$. The flow of intergranular liquid in the packed layer is a result of a balance between buoyancy forces, suction due to solidification shrinkage, and drag forces exerted by the matrix of packed grains. Inertial forces become negligible and in steady state the force balance can be approximated by the Darcy equation: $\left(g_{l}^{2} \mu_{l} / K\right)\left(\left\langle\overrightarrow{v_{l}}\right\rangle^{l}-\left\langle\overrightarrow{v_{s}}\right\rangle^{s}\right) \approx-g_{l} \nabla p+\rho_{l}^{b} g_{l} \vec{g}$. At low permeability, such as in the closely packed globular layer, the drag forces increase, making the buoyancy negligible. The percolation through the packed layer is then governed by a subtle interplay of drag and solidification shrinkage and its trajectory largely depends on the shape of the field of $g_{1}$ (the shape of the packed layer and the progression of solidification within). In a dendritic packed layer the permeability at the packing front is larger, which allows the buoyancy forces to exert an influence on the flow up to a certain depth into the packed layer. This modifies the trajectory of the liquid through the mushy zone and thus impacts the macrosegregation, generally diminishing the negative segregation in the center [1]. Our interpretations are based on simulations that use the same characteristic microstructure length for the permeability $\left(l_{\mathrm{c}}=75 \mu \mathrm{m}\right.$ in Eq. (11)) for globular and for dendritic packed layers. This is done due to lack of accurate information on the grain size and the dendrite spacing from the experiment. It is not clear whether this assumption is always reasonable. The characteristic length for permeability is often approximated by the secondary dendrite arm spacing (SDAS) in dendritic layers and by the grain size in globular layers. Dendritic grains are larger than globular grains, but if their SDAS is very fine (which is not the case for coarse dendrites often encountered in DC casting), the characteristic length for permeability in a dendritic packed layer can be smaller than that of the globular packed layer. Therefore, the permeability of a dendritic packed layer is not necessarily larger.

The effect of the packing fraction is similar for both grain morphologies (Fig. 10). An increase of the packing fraction leads to an amplification of the negative centerline segregation and to a shift of the positive segregation towards the billet surface. More extensive settling of solute-lean grains amplifies the negative segregation in the ingot center. In reaction, it enhances the positive segregation in the rest of the billet. However, due to the cylindrical geometry the amplification of the positive segregation is less marked in the concentration profile. The shift of the positive segregation towards the ingot surface is linked to the modification of the flow pattern. The raise of the packing front displaces the main flow loop towards the billet surface (Fig. 9a-b and c-d) and thus impacts the flow that is responsible for the macrosegregation formation.

The packing fraction impacts on the flow in two ways. First, the flow intensity increases due to an enhanced sedimentation of the solid grains, which drag along the liquid. When the packing fraction varies from 0.20 to 0.40 , the maximum liquid velocity increases from $1.2 \mathrm{~cm} / \mathrm{s}$ to $1.6 \mathrm{~cm} / \mathrm{s}$ for globular grains and from $0.09 \mathrm{~cm} / \mathrm{s}$ to $1.5 \mathrm{~cm} / \mathrm{s}$ for dendritic grains. The enhanced sedimentation is mainly due to a larger grain size. Because the grains grow up to a higher envelope fraction before packing, the equivalent grain diameter at packing increases from 125 to $150 \mu \mathrm{m}$ for globular grains and from 140 to $170 \mu \mathrm{m}$ for dendritic grains. Because the ratio of buoyancy to drag forces increases with the grain size, the settling velocity thus increases with the packing fraction. At the same equivalent diameter, the settling velocity of dendritic grains is smaller than that of globular grains due to the larger drag. The second effect of the grain packing fraction concerns the region where the liquid flow develops. Delaying the packing in terms of grain fraction does not imply a deepening of the sump as could possibly be expected. Instead, a global upward shift of the whole mushy zone occurs due to enhanced cooling and due to enhanced motion of grains to the center. The depth of both the mushy zone and of the liquid zone decrease. It is interesting to note that this decrease is more pronounced with globular 
grains. The reason is a larger enhancement of the heat transfer due to a more intense transport of the solid phase. Conversely, the decrease of the liquidus depth is stronger with dendritic grains, because a larger portion of the mushy zone is affected by the modification in the packing fraction (the grains globularize, this means that the increase of the envelope fraction is slower than the increase of the solid fraction).

Overall, the best agreement of the simulations with the experiment on the grain refined billet is obtained with the paraboloidal tip model (predicting globular grains) and with an envelope packing fraction of $g_{\mathrm{ep}}=0.20$. This holds for all compared characteristics: the segregation profile, the thermal field and the sump geometry. This packing fraction is smaller than the solid packing fraction of $g_{\mathrm{sp}}=$ 0.30 that was used in previous simulations of this billet [14]. Vreeman et al. commented that a better fit for the sump shape would be obtained with a solid packing fraction lower than 0.30 . Note that the packing fractions used in both model studies are much lower than the maximum value for random close packing of monodisperse spheres, which is around 0.65 [46,47]. Values of solid fraction at packing usually used in DC casting modeling range from 0.15 to $0.3[1,22,26,45,48,49]$. It is also interesting to note that in the $\mathrm{Al}-6 \mathrm{wt} \% \mathrm{Cu}$ alloy, the density of the liquid phase becomes higher than the solid density when the solid fraction increases above 0.4 . Thus the grains have the tendency of floating instead of settling. It is possible that the packing of the grains is affected by this inversion of the density difference.

\subsection{Influence of the amount of grain refiner on the macrosegregation and discussion on the non grain refined billet}

In DC casting, inoculants are usually added to the melt in order to refine the grain size. The absence of grain refinement affects the microstructure as well as the macrosegregation. Generally, experimental observations have shown an increase of grain size, a dendritization of the grain morphology and weaker macrosegregation at the centerline $[2,12,17,19]$. In the billet analyzed by Vreeman et al. [14], the removal of grain-refiner led to a slight decrease of macrosegregation. This can be seen by comparison of the experimental profiles shown in Figs. 10 and 11. Although characterizations of the microstructure have not been performed in the experiments, we can reasonably assume that in a non grain refined ingot the average grain size is larger and the grain morphology is more dendritic. The model should be able to reproduce such a transition of microstructure and macrosegregation between a refined and a non-refined casting.

The modeling of a non grain refined casting is not straightforward. Vreeman et al. assumed a fixed solid phase in their simulations of the non grain refined billet. As seen in Fig. 11, this strategy gave incorrect results. The assumption of a fixed solid would be adequate for a columnar microstructure. However, the measured macrosegregation profiles as well as other experiments on non grain refined DC castings $[12,18,50]$ indicate that the microstructure remains mostly equiaxed even in the absence of grain refinement. The present model is well adapted for describing heterogeneous athermal nucleation on inoculant particles with a known size distribution. In the absence of inoculant particles the population and the distribution of nucleation sites are not known. We tried to reproduce the influence of the grain refiner by altering the total population of the inoculant particles in the simulations. The nucleation was thus still described by the athermal heterogeneous nucleation model, but with a smaller number of nucleation sites. The level of refiner addition was varied by factors 10 with respect to the nominal refiner density of $N_{0}=1 \mathrm{~kg} / \mathrm{t}$. Four levels of grain refinement were simulated: $10 \mathrm{~kg} / \mathrm{t}\left(N_{0} \times 10\right), 1 \mathrm{~kg} / \mathrm{t}\left(N_{0}\right), 0.1 \mathrm{~kg} / \mathrm{t}\left(N_{0} / 10\right)$ and $0.01 \mathrm{~kg} / \mathrm{t}\left(N_{0} / 100\right)$. The same type of particle size distribution (as shown in Fig. 3) was used; only the population density of all inoculant 
particle classes was modified by the same factor. The envelope packing fraction was set to $g_{\text {ep }}=0.20$ in all simulations, since it gave the most accurate results for the grain-refined billet. The goal is not only to check whether the model is able to reproduce the experimentally observed macrosegregation in the non-refined billet, but also to analyze the effect of the refiner level on the structure.

Figs. $12 \mathrm{a}$ and $12 \mathrm{~b}$ provide information about the microstructure in the billet predicted by our model. They show the effect of the refiner addition level on the average grain size (represented by the mean final equivalent grain diameter, $\bar{d}_{s}$ ) and on the grain morphology (represented by the mean internal solid fraction at the packing front, $g_{\text {ip }}$ ), as predicted by both dendrite tip models. The mean final equivalent grain diameter is calculated as the equivalent grain diameter after complete solidification and by excluding the eutectic fraction:

$$
\bar{d}_{s}=\left(\frac{6\left(1-\bar{g}_{e u t}\right)}{\pi \bar{N}_{s}}\right)^{1 / 3}
$$

where $\bar{g}_{\text {eut }}$ is the mean eutectic volume fraction predicted by the model and $\bar{N}_{s}$ is the mean grain density. The effect of the inoculant density (grain refiner level) is similar for the two dendrite tip models: a reduction of the refiner level leads to an increase of the grain size and to a more dendritic morphology. On the opposite, an increase of the refiner level leads to smaller and more globular grains. However, important differences remain in the microstructure predictions, depending on the tip model used. The refiner level has a greater impact on the microstructure when the hemispherical model is considered, both in terms of grain size and morphology. With the hemispherical tip growth model, grains are already dendritic for the highest refiner level, and become even more dendritic when the refiner level is lowered. With the paraboloidal tip growth model, only the lowest refiner level leads to a significant reduction of the mean internal fraction. With higher refiner levels grains remain globular.
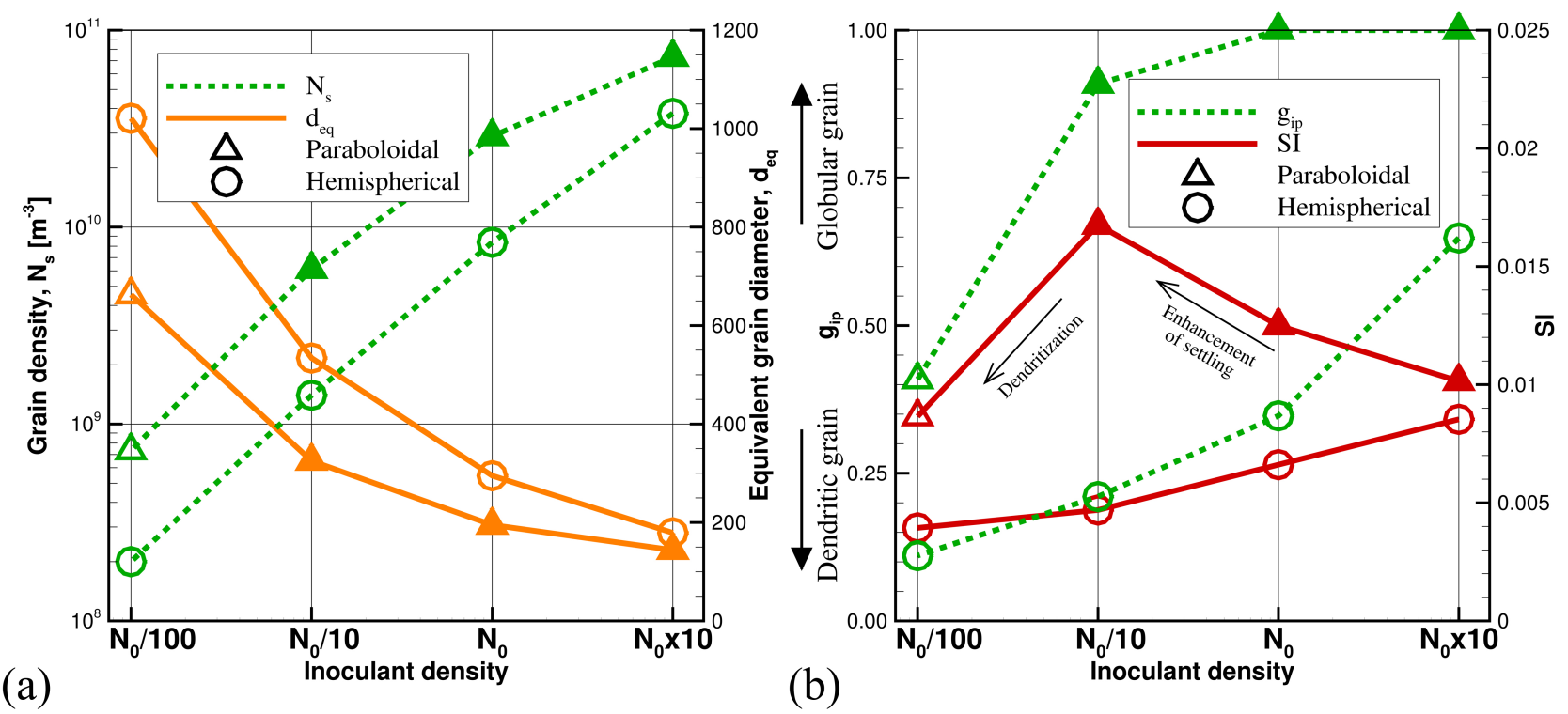

Figure 12: Microstructure and macrosegregation as a function of the nucleation density, predicted by both dendrite tip models. $N_{0}$ refers to the distribution of nucleation sites shown in Fig. 3. (a) Mean final grain density and mean equivalent grain diameter. (b) Mean internal solid fraction at packing $\left(g_{\mathrm{ip}}\right)$ and mean segregation index. Full symbols indicate globular and empty symbols dendritic grain morphology. 
Fig. 12a also shows the evolution of the mean final grain density as a function of the grain-refiner level. We can see that the dependence is not linear for either tip model. The nonlinearity is particularly strong with the paraboloidal model in the domain of globular grain morphologies (i.e. for inoculant densities of $N_{0} / 10$ and greater). This is a result of the complex coupling between the nucleation, the kinetics of grains growth and the transport of nucleant particles and grains [28,29,51]. When the number of nucleation sites is reduced, the consequence is that a smaller number of grains nucleate at a given undercooling. The rejection of solute into the liquid during their growth is less efficient and therefore the enrichment of the surrounding liquid is slower. The constitutional undercooling thus keeps increasing. As a consequence, nucleation sites requiring larger undercooling for their activation are activated. The reduction of the grain density is therefore not proportional to the reduction of the nuclei density but is less. This effect is stronger with globular grains due to the stronger coupling with grain transport [28].

The intensity of macrosegregation can be quantified in a compact form as a segregation index, SI. The segregation index is defined as the standard deviation of the macrosegregation across the billet radius:

$$
S I=\sqrt{\frac{2}{R_{b}^{2}} \int_{0}^{R_{b}}\left(C-C_{0}\right)^{2} r d r}
$$

The segregation indices for all simulations and their correlation with the grain morphology are shown in Fig. 12b. A comparison of the results obtained with both tip models indicates that the intensity of the macrosegregation is generally higher when the grains are more globular. This is further supported by the abrupt decrease of the SI upon the morphology transition predicted by the paraboloidal tip model. We can also see that with increasing grain size the SI increases, for globular grains whereas it decreases for dendritic grains.

We can take a closer look at what happens if we analyze the flow and the grain and solute transport in the sump. Fig. 13 shows the effect of the morphology transition on the macrosegregation. It shows the concentration field in the billet for nucleation-site densities of $N_{0} / 10$ and $N_{0} / 100$, corresponding to the transition from globular to dendritic morphology with the paraboloidal tip model. When the nucleation density is reduced from $N_{0}$ down to $N_{0} / 10$ (compare Fig. 9a to Fig. 13a), the segregation is enhanced everywhere in the billet. The main reason is that the grains, which remain globular, $\left(\bar{g}_{i p} \approx\right.$ $0.9)$ are almost twice larger. The effect of the grain settling is therefore increased. This is shown quantitatively in Fig. 14, where the difference of the velocity between the solid and the liquid in the slurry zone is plotted against the local grain size. We can see that across the whole range of nucleation densities the settling velocity globally increases with increasing grain size. When the grains are very small (around $80 \mu \mathrm{m}$ in Case $N_{0} \times 10$ ), their velocity is close to that of the liquid and thus macrosegregation is reduced. Larger grains settle faster, therefore the negative centerline macrosegregation increases when the nuclei density is reduced down to $N_{0} / 10$. When a transition to dendritic morphology $\left(\bar{g}_{i p} \approx 0.4\right)$ is triggered by further lowering the nuclei density to $N_{0} / 100$ (Fig. $13 b)$, the segregation is suddenly strongly reduced. It is interesting to note that the flow pattern remains very similar. Although the settling velocity further increases (Fig. 14), the expulsion of enriched liquid at the packing is lessened due to the smaller solid fraction at the packing front. This effect is shown in detail in Fig. 15 that displays the evolution of the mean concentration of copper and of the envelope and solid fractions along the billet centerline for cases with $N_{0} / 10$ (globular) and $N_{0} / 100$ (dendritic). We can see that with $N_{0} / 10$ (Fig. 15a) the intense settling of globular grains results into a large concentration jump and a negative centerline segregation $\left(C_{m} \approx 5.8\right)$ at the packing front. The negative segregation is then exacerbated mainly by the shrinkage flow through the packed layer. With $N_{0} / 100$ (Fig. 15b), the accumulation of dendritic grains at the packing front does not cause any 
603

604

605

606

significant segregation. The average concentration at the packing front is close to $6 \%$ - the nominal alloy concentration. The final negative centerline segregation is caused mainly by the shrinkage flow. This shows that the morphology transition reduces the influence of the grain packing on the macrosegregation pattern.

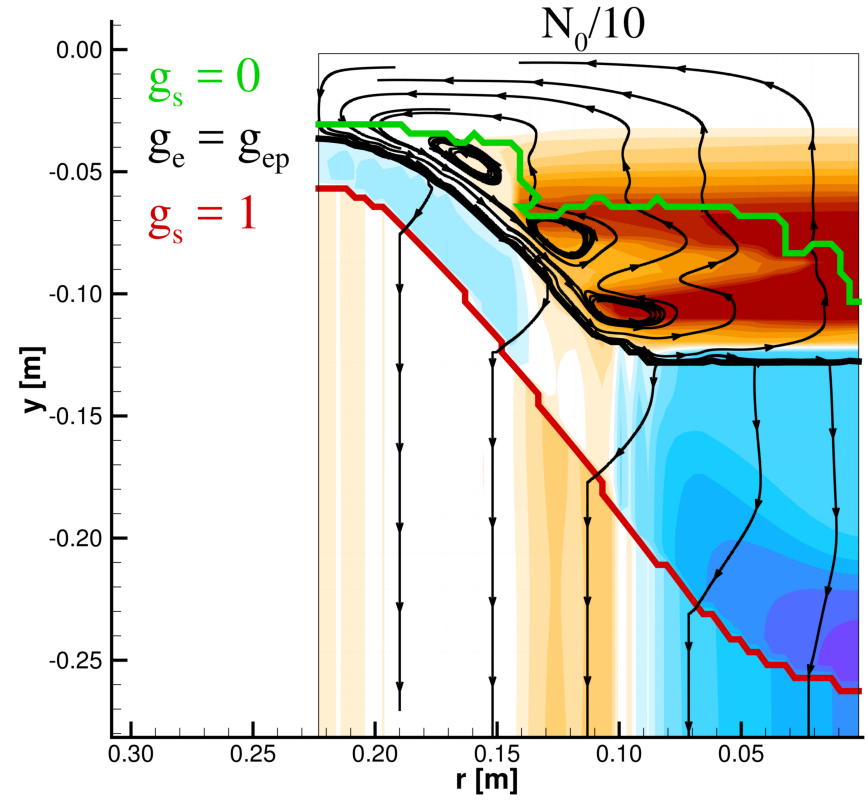

(a) Globular grains

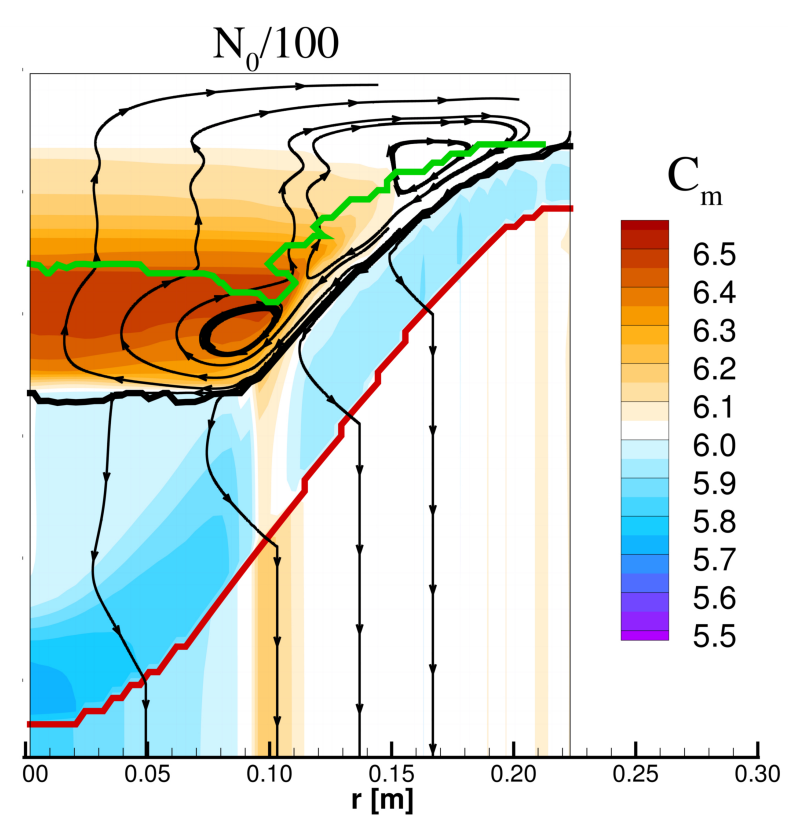

(b) Dendritic grains

Figure 13: The influence of the morphology transition on the macrosegregation. Fields of the average copper composition, (a) $N_{\text {ino }}=N_{0} / 10=0.1 \mathrm{~kg} / \mathrm{t}$, (b) $N_{\text {ino }}=N_{0} / 100=0.01 \mathrm{~kg} / \mathrm{t}$. Both simulations are with the paraboloidal dendrite tip model.

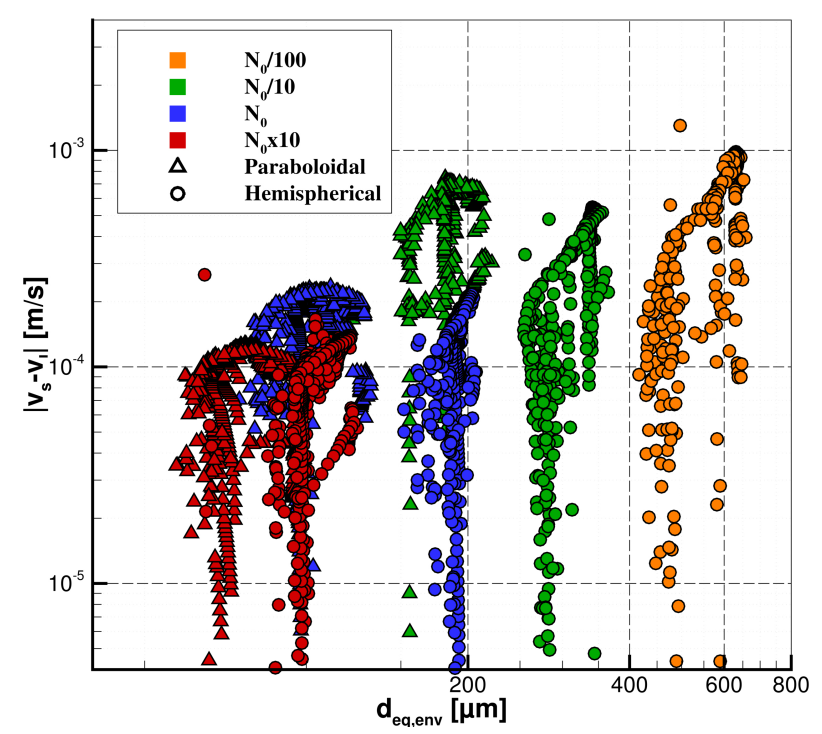

Figure 14: The slip velocity of the equiaxed grains in the slurry zone as a function of the local equivalent grain diameter, $d_{\mathrm{eq}, \mathrm{env}}$. Colored markers indicate a downward oriented slip velocity (settling) and (scarce) black markers indicate an upward oriented slip velocity (flotation). $N_{0}$ refers to the distribution of nucleation sites shown in Fig. 3. 


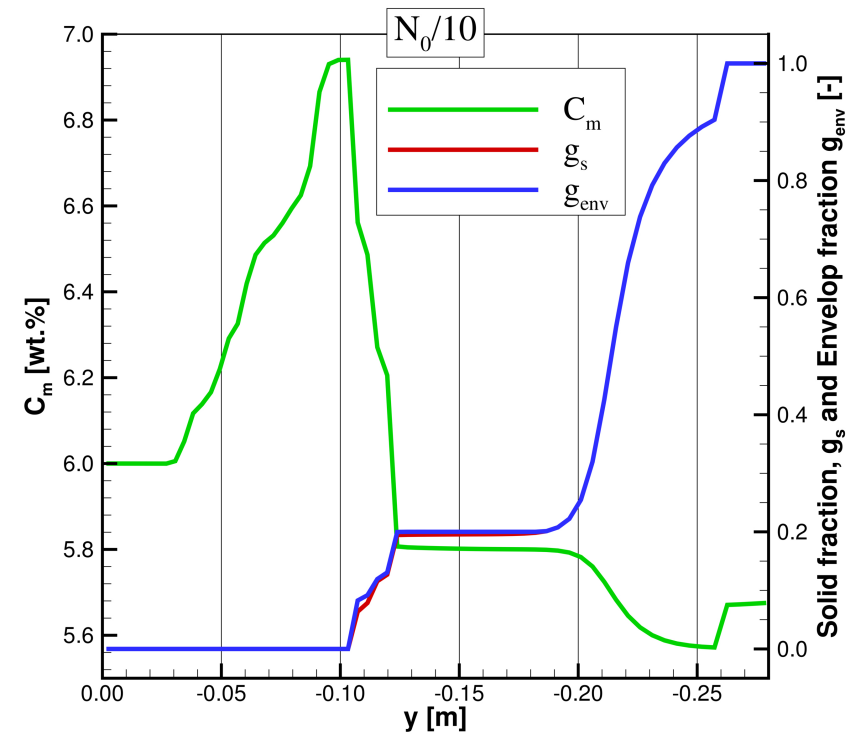

(a) Globular grains

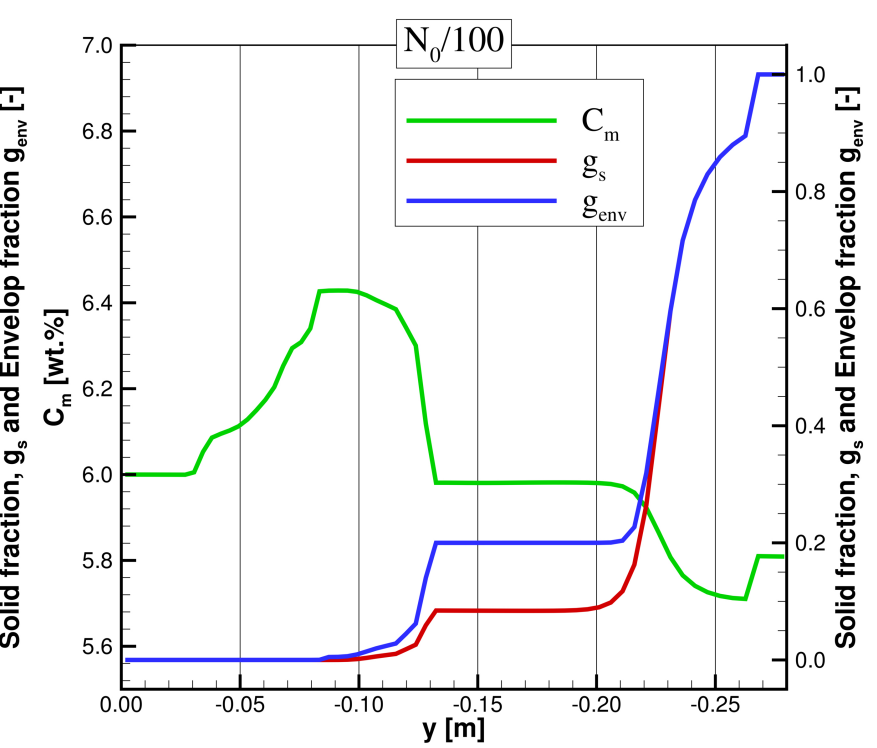

(b) Dendritic grains

Figure 15: The formation of the centerline macrosegregation with different grain morphologies. Evolution of the average copper composition $C_{\mathrm{m}}$, solid fraction $g_{\mathrm{s}}$ and envelope fraction $g_{\mathrm{e}}$ along the billet centerline for (a) $N_{0} / 10$ and (b) $N_{0} / 100$, both obtained with the paraboloidal tip model.

Macrosegregation profiles for all nucleation densities are presented in Fig. 11a, for the paraboloidal model and in Fig. 11b for the hemispherical model. The evolution of the profiles as a function of the nucleation density is consistent with the microstructure evolution shown in Fig. 12. With the paraboloidal dendrite tip model the grain size increases and the morphology remains globular for nucleation densities from $N_{0} \times 10$ down to $N_{0} / 10$. This leads to an amplification of the negative centerline macrosegregation. The segregation intensity is reduced only for the extreme case of $N_{0} / 100$, where the grains become dendritic. With the hemispherical dendrite tip model, the segregation intensity decreases all along as the density of nucleation sites is reduced. The main reason is the diminished grain settling due to the larger drag on the grains with a more pronounced dendritic morphology. In the extreme case of $N_{0} / 100$, the solid fraction at packing is only around $2 \%$.

Overall, our results indicate that the absence of grain refinement triggers a modification of the grain morphology from globular to dendritic. This morphology transition induces a weakening of the macrosegregation in the whole casting. The main reason is that the smaller solid fraction of the dendritic grains causes less expulsion of the solute-enriched liquid at the packing front. This trend is consistent with the experimental observations in the two billets and is predicted with a correct order of magnitude.

\section{Conclusions}

We performed a detailed study of the formation of macrosegregation in industrial size DC cast billets using a multiphase and multiscale model. This model stands out by the careful description of the intricate couplings between the kinetics of microstructure formation and the macroscopic transport of heat, mass, momentum and solute. It represents the nucleation, the growth kinetics and the transport of equiaxed grains; all taking into account the transitions between globular and dendritic grain morphologies. The model study was supported by previously published experimental results, which were used as a reference. 
632 Predictions of radial macrosegregation profiles, of lengthwise temperature profiles, and of the shape and the dimensions of the mushy zone were compared to the experimental measurements in a grain refined and a non grain refined billet. A sensitivity study with respect to the grain packing fraction was performed, in order to fit this poorly known but key model parameter. It showed that the rather low packing fraction of 0.2 clearly captures best both the heat transfer and the macrosegregation in the billets.

638

639

640

641

642

643

644

645

646

647

648

649

650

651

652

653

654

655

656

657

658

659

660

661

662

663

664

665

666

667

668

669

670

671

672

673

674

675

676

While the grain refiner addition level in the grain refined billet was known, the absence of grain refiner in the non grain refined billet was modeled by lowering the population density of nucleation sites. These simulations indicate that a morphology transition triggered by the grain refinement is the key to the explanation of the differences of the macrosegregation patterns in the two billets. In the grain refined billet, globular grain morphologies clearly gave better predictions of the thermal field and of the macrosegregation than dendritic morphologies. The reduction of the macrosegregation in the non grain refined billet was caused by a transition from globular to dendritic grain morphologies. It was shown that a globular grain morphology promotes the settling of equiaxed grains and thus exacerbates the associated transport of solute. It results into more pronounced negative centerline segregation than a dendritic grain morphology.

Previous work has shown that the choice of the dendrite tip model is not unambiguous when describing coarse dendritic morphologies. Two different dendrite tip models were therefore used to model the grain morphology and they gave disparate predictions. In the same solidification conditions, the hemispheric tip model tends to predict more dendritic grain morphologies than the paraboloidal tip model. The hemispheric tip model indicated dendritic grains in both the grain refined and the non grain refined billet, thus failing to capture a morphology transition and the associated reduction in macrosegregation. Only the paraboloid tip model was able to capture the transition from globular to dendritic grains as a function of grain refinement and to quantitatively reproduce its influence on the macrosegregation. A proper choice of the nucleation density was required, reducing the number of nucleation sites by around 100 times with respect to the grain refined case.

The formation of macrosegregation in DC casting is a result of a subtle interplay of several flow phenomena: thermosolutal convection, grain motion and packing, shrinkage-induced flow, and flow induced by thermomechanical deformation. The prominence of one or another of these phenomena may strongly depend on the casting size and shape (billet or ingot), on the alloy composition, on the mold type, and on the process parameters (casting speed, temperature, grain refinement). This work thus cannot provide a generally valid explanation of the macrosegregation mechanism in DC casting. It does however make a large step forward to a complete description of DC casting at the process scale, accounting for the coupling of microstructure and macrosegregation. Further work should focus on the refinement of the microstructure model, in order to ensure a quantitative prediction of the morphology transition between globular and dendritic grains. This is particularly important in the DC casting process, since the observed morphology of the grains is often coarse dendritic, i.e. close to the transition. A more extensive comparison of the models to well characterized industrial-scale experiments and application to multi-component alloys are also required.

\section{Acknowledgements}

This work was supported by the French National Research Agency (ANR) through the grant ANR2010-RNMP-007 (ANR Principia). It was also supported by the French State through the program "Investment in the future" operated by the ANR and referenced by ANR-11 LABX-0008-01 (LabEx DAMAS). 
L. Heyvaert et al., Coupling of microstructure and macrosegregation ..., Metall. Mater. Trans. A 48 (2017)

\section{References}

[1] M. Založnik, A. Kumar, H. Combeau, M. Bedel, P. Jarry, and E. Waz: Adv. Eng. Mater., 2011, vol. 13, pp. 570-80.

[2] R. Nadella, D.G. Eskin, Q. Du, and L. Katgerman: Prog. Mater. Sci., 2008, vol. 53, pp. 42180.

[3] D.G. Eskin, R. Nadella, and L. Katgerman: Acta Mater., 2008, vol. 56, pp. 1358-65.

[4] L.C. Nicolli, A. Mo, and M. M'Hamdi: Metall. Mater. Trans. A, 2005, vol. 36, pp. 433-42.

[5] S.R. Wagstaff and A. Allanore: Metall. Mater. Trans. B, 2016, vol. 47, pp. 1-7.

[6] H. Combeau, M. Založnik, S. Hans, and P.E. Richy: Metall. Mater. Trans. B, 2009, vol. 40, pp. 289-304.

[7] M. Založnik and H. Combeau: in Model. Cast. Weld. Adv. Solidif. Process. XII, Steve L Cockroft and Daan M Maijer, eds., TMS, Warrendale (PA), USA, 2009, pp. 165-72.

[8] A. Kumar, M. Založnik, and H. Combeau: Int. J. Adv. Eng. Sci. Appl. Math., 2011, vol. 2, pp. $140-48$.

[9] N. Leriche, H. Combeau, C.-A. Gandin, and M. Založnik: in Model. Cast. Weld. Adv. Solidif. Process. XIV, Hideyuki Yasuda, Hervé Combeau, and Charles A Monroe, eds., Awaji, Japan, 2015.

[10] H. Combeau, M. Založnik, and M. Bedel: JOM, 2016, vol. 68, pp. 2198-2206.

[11] D.G. Eskin, V.I. Savran, and L. Katgerman: Metall. Mater. Trans. A, 2005, vol. 36A, pp. 1965-76.

[12] R. Nadella, D.G. Eskin, and L. Katgerman: Metall. Mater. Trans. A, 2008, vol. 39, pp. 45061.

[13] T.L. Finn, M.G. Chu, and W.D. Bennon: in Micro/Macro Scale Phenom. Solidif., Christoph Beckermann, L A Bertram, S J Pien, and R E Smelser, eds., ASME, New York, 1992, pp. 17-24.

[14] C.J. Vreeman, J.D. Schloz, and M.J.M. Krane: J. Heat Transfer, 2002, vol. 124, p. 947.

[15] B. Gariepy and Y. Caron: in Light Met. 1991, Elwin Rooy, ed., TMS, Warrendale (PA), USA, 1991, pp. 961-71.

[16] A. Joly, G.-U. Grün, D. Daloz, H. Combeau, and G. Lesoult: Mater. Sci. Forum, 2000, vol. 329-330, pp. 111-20.

[17] G. Lesoult, V. Albert, B. Appolaire, H. Combeau, D. Daloz, A. Joly, C. Stomp, G.-U. Grün, and P. Jarry: Sci. Technol. Adv. Mater., 2001, vol. 2, pp. 285-91.

[18] D. Daloz, H. Combeau, A. Joly, G. Lesoult, G.-U. Grün, P. Jarry, and B. Commet: in Mater. 2002 La Concept. À La Mise En Cuvre, Tours, France, 2002.

[19] A.M. Glenn, S.P. Russo, and P.J.K. Paterson: Metall. Mater. Trans. A, 2003, vol. 34, pp. 1513-23.

[20] A. V Reddy and C. Beckermann: Metall. Mater. Trans. B, 1997, vol. 28B, pp. 479-89.

[21] T. Jalanti, M. Swierkosz, M. Gremaud, and M. Rappaz: in DGM Conf., Frankfurt, Germany, 2000.

[22] C.J. Vreeman, M.J.M. Krane, and F.P. Incropera: Int. J. Heat Mass Transf., 2000, vol. 43, 
L. Heyvaert et al., Coupling of microstructure and macrosegregation ..., Metall. Mater. Trans. A 48 (2017)

pp. 677-86.

[23] M. Založnik and B. Šarler: Mater. Sci. Eng. A, 2005, vol. 413-414, pp. 85-91.

[24] L. Zhang, D.G. Eskin, A. Miroux, T. Subroto, and L. Katgerman: Metall. Mater. Trans. B, 2012, pp. 1-9.

[25] Q. Du, D.G. Eskin, and L. Katgerman: Metall. Mater. Trans. A, 2007, vol. 38, pp. 180-89.

[26] A. V Reddy and C. Beckermann: in Mater. Process. Comput. Age II, Vaughan R Voller, Steve P Marsh, and Nagy El-Kaddah, eds., TMS, 1995, pp. 89-102.

[27] A. Håkonsen, D. Mortensen, S. Benum, and H.E. Vatne: in Light Met. 1999, Edward C. Eckert, ed., TMS, Warrendale (PA), USA, 1999, pp. 821-27.

[28] M. Bedel, K.O. Tveito, M. Založnik, H. Combeau, and M. M'Hamdi: Comput. Mater. Sci., 2015, vol. 102, pp. 95-109.

[29] M. Bedel, L. Heyvaert, M. Založnik, H. Combeau, D. Daloz, and G. Lesoult: IOP Conf. Ser. Mater. Sci. Eng., 2015, vol. 84, p. 12100.

[30] C.Y. Wang and C. Beckermann: Metall. Mater. Trans. A, 1996, vol. 27, pp. 2754-64.

[31] B. Appolaire, H. Combeau, and G. Lesoult: Mater. Sci. Eng. A, 2008, vol. 487, pp. 33-45.

[32] M. Založnik and H. Combeau: Comput. Mater. Sci., 2010, vol. 48, pp. 1-10.

[33] A.L. Greer, A.M. Bunn, A. Tronche, P. V Evans, and D.J. Bristow: Acta Mater., 2000, vol. 48, pp. 2823-35.

[34] M. Rappaz and P. Thévoz: Acta Metall., 1987, vol. 35, pp. 1487-97.

[35] K.O. Tveito: NTNU, Trondheim, Norway, 2017.

[36] J. Lipton, M.E. Glicksman, and W. Kurz: Metall. Trans. A, 1987, vol. 18, pp. 341-45.

[37] W. Kurz and D.J. Fisher: Fundamentals of Solidification, Trans Tech Publications, Aedermannsdorf, Switzerland, 1998.

[38] Ø. Nielsen, B. Appolaire, H. Combeau, and A. Mo: Metall. Mater. Trans. A, 2001, vol. 32, pp. 2049-60.

[39] M. Ishii: Thermo-Flud Dynamic Theory of Two-Phase Flow, Eyerolles, Paris, 1975.

[40] J. Ni and C. Beckermann: J. Mater. Process. Manuf. Sci., 1993, vol. 2, pp. 217-31.

[41] G.H. Yeoh and J. Tu: Computational Techniques for Multiphase Flows, ButterworthHeinemann, 2009.

[42] C.J. Vreeman, M.J.M. Krane, and J.D. Schloz: in Comput. Model. Mater. Miner. Met. Process., M Cross, J W Evans, and C Bailey, eds., TMS, Warrendale (PA), USA, 2001, pp. 397-409.

[43] D.C. Weckman and P. Niessen: J. Electron. Mater., 1992, vol. 21, pp. 593-602.

[44] A. Tronche: University of Cambridge, 2000.

[45] M. Založnik, A. Kumar, H. Combeau, M. Bedel, P. Jarry, and E. Waz: in Essent. Readings Light Met. Vol. 3, Cast Shop Alum. Prod., John F Grandfield and Dmitry G Eskin, eds., John Wiley \& Sons, Hoboken (NJ), USA, 2013, pp. 848-53.

[46] C. Lesaffre, V. Mineau, D. Picart, and H. Van Damme: Comptes Rendus l'Académie Des Sci. - Ser. IV - Phys., 2000, vol. 1, pp. 647-53.

[47] C. Song, P. Wang, and H.A. Makse: Nature, 2008, vol. 453, pp. 629-32. 
757 [48] I. Vušanović and M.J.M. Krane: IOP Conf. Ser. Mater. Sci. Eng., 2012, vol. 27, p. 12069.

758 [49] K. Fezi, J. Coleman, and M.J.M. Krane: in Light Met. 2015, Margaret Hyland, ed., TMS, 759 Warrendale (PA), USA, 2015, pp. 871-75.

760 [50] R. Nadella, D.G. Eskin, and L. Katgerman: Mater. Sci. Forum, 2006, vol. 519-521, $761 \quad$ pp. 1841-46.

762 [51] M. Bedel: Université de Lorraine, Nancy, France, 2014.

763 [52] K.O. Tveito, M. Bedel, M. Založnik, H. Combeau, and M. M'Hamdi: IOP Conf. Ser. Mater. 764 Sci. Eng., 2012, vol. 27, p. 12040.

765 [53] R. Ananth and W.N. Gill: J. Cryst. Growth, 1991, vol. 108, pp. 173-89. 


\section{Appendix A}

In the three-phase solidification model, the growth kinetics of the equiaxed grains is described by solute balances over the three hydrodynamic phases and on two moving interfaces, the solidinterdendritic-liquid interface and the envelope-extradendritic-liquid interface. The volume averaged mass transfer rate per unit volume from the interdendritic liquid to the solid due to phase change at the solid-liquid interface is denoted $\Gamma_{s}$. The mass transfer rate from the extradendritic liquid to the envelope at the envelope-liquid interface is $\Gamma_{e}$. The solid-liquid interface is at equilibrium and the equilibrium concentrations of solid and liquid are linked by the partition coefficient: $C_{s}^{*}=k C_{l}^{*}$. The interdendritic liquid is also entirely at the liquid equilibrium concentration $C_{l}^{*}$. The solute transfer rates due to this interfacial growth are $\Gamma_{s} C_{s}^{*}$ and $-\Gamma_{S} C_{l}^{*}$ at the solid and liquid sides of the solid-liquid interface, and $\Gamma_{e} C_{l}^{*}$ and $-\Gamma_{e} C_{l}^{*}$ at the exterior and the interior sides of the envelope-liquid interface, respectively. The diffusion fluxes from the solid-liquid interface into the solid phase, $j_{s}^{d}$, and from the envelope into the extradendritic liquid, $j_{l}^{d}$, are modeled as:

$$
j_{s}^{d}=\frac{\rho_{s} S_{v}^{s} D_{s}}{\delta_{s}}\left(C_{s}^{*}-\left\langle C_{s}\right\rangle^{c}\right) \quad j_{l}^{d}=\frac{\rho_{l} S_{v}^{e} D_{l}}{\delta_{l e}}\left(C_{l}^{*}-\left\langle C_{l}\right\rangle^{e}\right)
$$

The diffusion length in the solid is $\delta_{s}=d_{s} / 10$, where $d_{s}$ is the equivalent diameter of the solid. The diffusion length in the liquid, $\delta_{e}$, is modeled by an expression that accounts for the effects of liquid convection and of a moving interface and was derived in [52]. The diffusion fluxes from both interfaces into the interdendritic phase, $j_{i s}^{d}$ and $j_{i e}^{d}$, become indeterminate under the assumption of infinite diffusion in the extradendritic liquid. The solute balance at the solid-interdendritic-liquid interface is

$$
\Gamma_{s} C_{s}^{*}+\frac{\rho_{s} S_{v}^{s} D_{s}}{\delta_{s}}\left(C_{s}^{*}-\left\langle C_{s}\right\rangle^{s}\right)=\Gamma_{s} C_{l}^{*}-j_{i s}^{d}
$$

The solute balance at the envelope-extradendritic-liquid interface is

$$
\Gamma_{e} C_{l}^{*}+j_{i e}^{d}=\Gamma_{e} C_{l}^{*}-\frac{\rho_{l} S_{v}^{e} D_{l}}{\delta_{e}}\left(C_{l}^{*}-\left\langle C_{l}\right\rangle^{l e}\right)
$$

To avoid the indeterminate terms we can write down the sum of both interfacial balances as the solute balance for the interdendritic phase [35]:

$$
\frac{\partial}{\partial t}\left(\rho_{l} g_{l i} C_{l}^{*}\right)=\Gamma_{e} C_{l}^{*}-\Gamma_{s} C_{s}^{*}-\frac{\rho_{l} S_{v}^{e} D_{l}}{\delta_{e}}\left(C_{l}^{*}-\left\langle C_{l}\right\rangle^{l e}\right)-\frac{\rho_{s} S_{v}^{s} D_{s}}{\delta_{s}}\left(C_{s}^{*}-\left\langle C_{s}\right\rangle^{s}\right) .
$$

We take into account that $g_{l i}=g_{e}-g_{s}$ and $g_{l}=1-g_{s}$, that $g_{l}\left\langle C_{l}\right\rangle^{l}=g_{l e}\left\langle C_{l}\right\rangle^{l e}+g_{l i} C_{l}^{*}$ and we obtain

$$
\frac{\rho_{s} S_{v}^{S} D_{s}}{\delta_{s}}\left(k C_{l}^{*}-\left\langle C_{s}\right\rangle^{s}\right)+\left(\frac{1-g_{s}}{1-g_{e}}\right) \frac{\rho_{l} S_{v}^{S} D_{l}}{\delta_{e}}\left(C_{l}^{*}-\left\langle C_{l}\right\rangle^{l}\right)=\Gamma_{s}(1-k) C_{l}^{*}-\rho_{l}\left(g_{e}-g_{s}\right) \frac{\partial C_{l}^{*}}{\partial t} .
$$

This equation is solved in conjunction with:

- the heat conservation equation that gives the evolution of the local average enthalpy, defined as $\bar{h}=c_{p} T+\left(1-g_{s}\right) L_{f}$;

- the phase diagram that gives the link between the equilibrium liquid concentration and the temperature: $T=T_{m}+m C_{l}^{*}$;

- and a dendrite-tip model that gives the velocity of the envelope growth as a function of the local supersaturation $\left(C_{l}^{*}-\left\langle C_{l}\right\rangle^{l e}\right)$ and thus enables us to calculate the envelope fraction $g_{e}$. This gives four equations for the four additional unknowns $\Gamma_{s}, C_{l}^{*}, T$ and $g_{e}$.

Several supplementary geometric relations are required. The grain envelopes are assumed to be regular octahedra, the distance between the center and the vertices of the octahedron corresponding 
802

803

804

\section{5}

806

807

808

809 The interpolation is done by the internal solid fraction, $g_{i}=g_{s} / g_{e}$, and the exponent $m$ was set to 6 810 [38]. The secondary dendrite arm spacing is approximated as be a fraction of the envelope radius: $811 \quad \lambda_{2}=R_{e} / 5=R_{\text {arm }} /\left(5 \pi^{1 / 3}\right)$.

to the length of the primary dendrite arms. The primary arm length, $R_{\text {arm }}$, is calculated by integrating the tip velocity over time. Knowing the primary arm length, the envelope fraction and the specific surface of the envelope are:

$$
g_{e}=\frac{4}{3} N_{v} R_{\text {arm }}^{3} \text { and } S_{v}^{e}=4 \sqrt{3} R_{\text {arm }}^{2} N_{v}
$$

where $N_{v}$ is the grain population density. The specific surface of the solid, $S_{v}^{s}$, is described by a generalized correlation that continuously switches between a relation for a strongly dendritic grain, in which the surface area is controlled by the secondary dendrite arm spacing, $\lambda_{2}$, and a relation for a globular grain, where $S_{v}^{s}=S_{v}^{e}[31]$ :

$$
S_{v}^{s}=\frac{4}{\lambda_{2}} g_{i}^{1 / 2} g_{e}\left(1-g_{i}^{m}\right)+g_{i}^{m} S_{v}^{e}
$$


L. Heyvaert et al., Coupling of microstructure and macrosegregation ..., Metall. Mater. Trans. A 48 (2017)

\section{Figure captions}

815

Figure 1: (a) Schematics of the hydrodynamic phases in the three-phase model. (b) Solute profiles in the three phases, solute concentrations and volume fractions.

816

Figure 2: Evolution of solid, liquid and average densities of the alloy Al-6wt.\%Cu with solid fraction. The solidification path is assumed to follow the Scheil-Gulliver model. The variation of the solid density due to the appearance of a secondary phase upon the eutectic reaction is not accounted for.

Figure 3: Discretization of the distribution of nucleation sites with respect to the critical undercooling. The distribution corresponds to the size distribution of TiB $B_{2}$ particles for $1 \mathrm{~kg} / \mathrm{t}$ of Al5 Ti-1B grain refiner [28,44].

Figure 4: Heat transfer and flow in the billet: (a) temperature field, (b) solid fraction, (c) norm of the liquid velocity and liquid velocity streamlines, (d) norm of the solid velocity and solid velocity streamlines. The grain refined billet simulated with a packing fraction of $g_{e p}=0.2$ and the paraboloidal dendrite tip model is shown. The relative velocities are shown in the reference frame of the moving product.

Figure 5: Internal solid fraction in the mushy zone obtained with both dendrite tip models: (a) paraboloidal, (b) hemispherical tip model. The packing fraction is $g_{e p}=0.20$ in both cases.

Figure 6: Internal solid fraction at packing $\left(g_{\mathrm{ip}}\right)$ for both tip growth models, hemispherical (solid lines) and paraboloidal (dashed lines).

Figure 7: Comparison of measured and computed vertical temperature profiles at three different radius in the ingot (subsurface in blue, midradius in green and centerline in red). (a) Comparison of simulations with globular grains (paraboloidal tip model) and $g_{e p}=0.2$ with experiments and with simulations of Vreeman et al. [14]. (b) Influence of the packing fraction on the temperature profiles for globular grain morphology (paraboloidal tip model). (c) Influence of the packing fraction on the temperature profiles for dendritic grain morphology (hemispherical tip model).

Figure 8: (a) Comparison of the sump shape obtained with the paraboloidal tip growth model and $g_{e p}=0.20$ to the experimental sump shape. (b) Relative error of the lengths L1 and L2 as a function of the packing fraction, shown for simulations with both tip models.

Figure 9: Macrosegregation of copper in the billet. Fields of average concentration for packing fractions of $g_{e p}=0.20$ and $g_{e p}=0.40$ and both dendrite tip models: (a) paraboloidal tip model and (b) hemispherical tip model. 
Figure 10: Macrosegregation in the grain-refined billet. Profiles of copper concentration from simulations with different packing fractions and both dendrite tip models: (a) paraboloidal tip model and (b) hemispherical tip model. The model predictions are compared to the experimental profiles and to simulations of Vreeman et al. [14].

Figure 11: Macrosegregation in the non grain-refined billet. Profiles of copper concentration from simulations with different nucleation densities and both dendrite tip models: (a) paraboloidal tip model and (b) hemispherical tip model. The model predictions are compared to the experimental profiles and to simulations of Vreeman et al. [14]. $N_{0}$ refers to the distribution of nucleation sites shown in Fig. 3.

Figure 12: Microstructure and macrosegregation as a function of the nucleation density, predicted by both dendrite tip models. $N_{0}$ refers to the distribution of nucleation sites shown in Fig. 3. (a) Mean final grain density and mean equivalent grain diameter. (b) Mean internal solid fraction at packing $\left(g_{\mathrm{ip}}\right)$ and mean segregation index. Full symbols indicate globular and empty symbols dendritic grain morphology.

Figure 13: The influence of the morphology transition on the macrosegregation. Fields of the average copper composition, (a) $N_{\text {ino }}=N_{0} / 10=0.1 \mathrm{~kg} / \mathrm{t}$, (b) $N_{\text {ino }}=N_{0} / 100=0.01 \mathrm{~kg} / \mathrm{t}$. Both simulations are with the paraboloidal dendrite tip model.

Figure 14: The slip velocity of the equiaxed grains in the slurry zone as a function of the local equivalent grain diameter, $d_{\mathrm{eq}, \mathrm{env}}$. Colored markers indicate a downward oriented slip velocity (settling) and (scarce) black markers indicate an upward oriented slip velocity (flotation). $N_{0}$ refers to the distribution of nucleation sites shown in Fig. 3.

Figure 15: The formation of the centerline macrosegregation with different grain morphologies. Evolution of the average copper composition $C_{\mathrm{m}}$, solid fraction $g_{\mathrm{s}}$ and envelope fraction $g_{\mathrm{e}}$ along the billet centerline for (a) $N_{0} / 10$ and (b) $N_{0} / 100$, both obtained with the paraboloidal tip model. 
L. Heyvaert et al., Coupling of microstructure and macrosegregation ..., Metall. Mater. Trans. A 48 (2017)

\section{Table captions}

830

Table 1: Summary of the model equations

Table 2: Thermo-physical properties and model parameters

831 
832 Nomenclature

\section{Latin symbols}

$C_{0}$

$C_{d}$

$C_{k}^{*}$

$\left\langle C_{k}\right\rangle^{k}$

$C_{m}$

$c_{p}$

$C_{\text {ref }}$

$d_{g}$

$D_{k}$

$d_{s}$

$\bar{d}_{s}$

$\vec{g}$

$g_{e p}$

$g_{e p}^{\max }$

$\bar{g}_{\text {eut }}$

$g_{i}$

$g_{i p}$

$g_{k}$

$h_{k}$

$j_{k}^{d}$

$k$

K

$k_{k}$

$L_{1}$

$L_{2}$

$l_{c}$

$L_{f}$

m

$M_{k}^{d}$

$M_{k}^{\Gamma}$

$M_{k}^{\Phi}$
Nominal solute concentration

Drag coefficient

Solute concentration at the solid-liquid interface in phase $\mathrm{k}$

Average solute concentration in phase $\mathrm{k}$

Average "mixture" concentration

Specific heat

Reference solute concentration for the liquid density

Equivalent grain envelope diameter

Solute diffusion coefficient in phase $\mathrm{k}$

Equivalent diameter of the solid phase

Mean final equivalent grain diameter

Gravity acceleration

Packing grain-volume fraction

Maximum envelope packing fraction

Mean final eutectic volume fraction

Internal solid volume fraction

Mean internal solid volume fraction at the packing front

Volume fraction of phase $\mathrm{k}$

Mass enthalpy of phase $\mathrm{k}$

Diffusion flux from the solid-liquid interface into phase $\mathrm{k}$

Equilibrium partition coefficient

Permeability

Kozeny constant

Distance between the packing front and the solidus at mid-radius

Height of the solidus front

Characteristic length of the porous microstructure

Latent heat

Liquidus slope

Interfacial drag force on phase $\mathrm{k}$ per unit volume

Momentum flux to phase $\mathrm{k}$ due to phase change

Momentum transfer to phase $\mathrm{k}$ due to nucleation 
$N_{0} \quad$ Nominal population density of grain refiner particles

$N_{g} \quad$ Grain density (number of grains per unit volume)

$N_{\text {nuc }}^{i} \quad$ Density of $i^{\text {th }}$ class of inoculant particles

$\bar{N}_{s} \quad$ Mean final grain density

$p \quad$ Pressure

$P e_{C}, P e_{T} \quad$ Solutal, thermal Péclet nuber of the dendrite tip related to growth

$\mathrm{Pr} \quad$ Prandtl number

$P u_{C}, P u_{T} \quad$ Solutal, thermal Péclet nuber of the dendrite tip related to convection

$R_{\text {arm }} \quad$ Primary dendrite arm length

$R_{b} \quad$ Billet radius

$R e_{C}, R e_{T} \quad$ Solutal, thermal Reynolds number

$R_{\text {tip }} \quad$ Dendrite tip radius

Sc Schmidt number

SDAS Secondary dendrite arm spacing

$S I$

Segregation index

$S_{v}^{e}$

Specific surface of the dendrite envelope

$S_{v}^{S}$

Specific surface of solid phase

$t$

Time

$T$

Temperature

$T_{m}$

Melting temperature of pure aluminum

$T_{\text {ref }}$

Reference temperature for the liquid density

$\Delta T$

Undercooling

$V_{0}$

Initial grain volume at nucleation

$\left\langle\overrightarrow{v_{k}}\right\rangle^{k}$

Intrinsic average velocity of phase $\mathrm{k}$

$V_{\text {tip }}$

Dendrite tip velocity 


\section{Greek symbols}

$\alpha$

$\beta_{C}$

$\beta_{T}$

$\beta_{s l}$

$\Gamma_{k}$

$\Gamma_{\mathrm{GT}}$

$\delta$

$\delta_{e}$

$\delta_{s}$

$\lambda$

$\lambda_{2}$

$\mu_{k}$

$\rho_{k}$

$\rho_{l}^{b}$

$\rho_{\text {ref }}$

$\sigma^{*}$

$\tau$

$\left\langle\tau_{k}\right\rangle^{k}$

$\Phi_{k}$

$\phi_{k}$

$\Omega_{C}$

$\Omega_{T}$

Thermal diffusivity

Solutal expansion coefficient for the liquid density

Thermal expansion coefficient for the liquid density

Constant solidification shrinkage coefficient

Mass flux into phase $\mathrm{k}$ per unit volume at the interface due to phase change

Gibbs-Thomson coefficient

Dirac delta function

Solute diffusion length in the extra-dendritic liquid phase

Solute diffusion length in the solid phase

Thermal conductivity

Secondary dendrite arm spacing

Dynamic viscosity of phase $\mathrm{k}$

Density of phase $\mathrm{k}$

Liquid density in the buoyancy term

Reference density for the buoyancy term

Tip selection constant

Tortuosity of the porous medium

Shear stress tensor in phase $\mathrm{k}$

Mass flux into phase $\mathrm{k}$ per unit volume due to nucleation

Mass flux due to nucleation

Dimensionless supersaturation

Dimensionless undercooling

835

\section{Sub- and superscripts}

e

1

le

li

$\mathrm{S}$

Envelope

Liquid

Extradendritic liquid

Intradendritic liquid

Solid 